Analysis of edge and surface TCTs for irradiated 3D silicon strip detectors

This content has been downloaded from IOPscience. Please scroll down to see the full text. 2013 JINST 8 P03002

(http://iopscience.iop.org/1748-0221/8/03/P03002)

View the table of contents for this issue, or go to the journal homepage for more

Download details:

IP Address: 128.240.233.146

This content was downloaded on $21 / 11 / 2016$ at $04: 31$

Please note that terms and conditions apply.

You may also be interested in:

TCT measurements of irradiated strip detectors with a focused laser beam

I Mandi, V Cindro, A Gorišek et al.

Simulation and laboratory test results of 3D CMS pixel detectors for HL-LHC

E Alagoz, M Bubna, A Krzywda et al.

Design, fabrication and characterization of the first dual-column 3D stripixel detectors

D Bassignana, Z Li, M Lozano et al.

Planar pixel sensors for the ATLAS upgrade: beam tests results

$\mathrm{J}$ Weingarten, S Altenheiner, M Beimforde et al.

Modeling of electric field in silicon micro-strip detectors irradiated with neutrons and pions

G. Kramberger, V. Cindro, I. Mandi et al.

Prototype ATLAS IBL modules using the FE-14A front-end readout chip

The ATLAS IBL collaboration

Operational voltage of silicon heavily irradiated strip detectors utilizing avalanche

multiplication effect

E Verbitskaya, V Eremin and A Zabrodskii 


\section{Analysis of edge and surface TCTs for irradiated 3D silicon strip detectors}

\section{G. Stewart, ${ }^{a, 1}$ R. Bates, ${ }^{a}$ C. Fleta, ${ }^{b}$ G. Kramberger,${ }^{c}$ M. Lozano, ${ }^{b}$ M. Milovanovic ${ }^{c}$} and G. Pellegrini ${ }^{b}$

${ }^{a}$ School of Physics and Astronomy, University of Glasgow,

University Avenue, Glasgow, U.K.

${ }^{b}$ Department of Experimental Particle Physics, Jozef Stefan Institute, Jamova cesta, Ljubljana, Slovenia

${ }^{c}$ Centro Nacional de Microelectrónica, Campus Universidad Autónoma de Barcelona,

Carrer Universitat Autonoma B, Barcelona, Spain

E-mail: gd.stewart@physics.gla.ac.uk

\footnotetext{
${ }^{1}$ Corresponding author.
} 
ABstRaCT: We performed edge and surface Transient Current Technique (TCT) measurements of short, double sided 3D silicon strip detectors. Double sided 3D devices are a useful counterpart to traditional planar devices for use in the highest radiation environments. The TCT technique allows the electric field in the 3D devices to be probed in a way not possible before. The TCT technique uses the current waveform produced by the detector in response to a near delta function point laser pulse (illumination). The waveforms are recorded as a function of illumination position over the surface of the device under test as a function of detector bias.

This data gives information on the portion of the induced signal from electron or hole motion. From the rise times of the signals the velocity profile of the carriers in the devices and therefore electric fields can be determined. The collected charge was calculated from the integral of the waveforms.

The detectors were tested prior to irradiation, after irradiating to a dose of $5 \times 10^{15} 1 \mathrm{MeV}$ equivalent neutrons $/ \mathrm{cm}^{2}$, and after periods of annealing at elevated temperatures. Annealing was achieved in situ by warming to $60^{\circ} \mathrm{C}$ for 20 to 600 minutes corresponding to room temperature annealing of between 8 and 200 days.

While before irradiation, full lateral depletion between the columns occurs at low bias voltages, at approximately $3 \mathrm{~V}$, a uniform carrier velocity between the columns is not achieved until $40 \mathrm{~V}$. Both the drift of electrons and holes provide equal contributions to the measured signals. After irradiation there is clear charge multiplication enhancement along the line between columns with a very non-uniform velocity profile in the unit cell of the device. In addition, charge trapping greatly suppresses the contribution of the holes on the signal produced.

KEYWORDS: Radiation-hard detectors; Charge transport and multiplication in solid media; Charge induction; Radiation damage to detector materials (solid state) 


\section{Contents}

1 Introduction and methods $\quad 1$

2 Results 3

2.1 CV and IV characteristics 3

2.2 Edge TCTs for non-irradiated sensors 3

2.3 Spatially resolved top surface TCTs for non-irradiated sensors 9

$\begin{array}{lll}2.4 & \text { Surface TCTs for irradiated sensors } & 13\end{array}$

$\begin{array}{lll}2.5 & \text { Surface TCTs for irradiated sensors post-annealing } & 16\end{array}$

3 Conclusions $\quad 20$

\section{Introduction and methods}

We performed spatially-resolved edge and surface Transient Current Technique (TCT) measurements of a double sided 3D silicon strip detector. TCT measurements of silicon devices have been performed since the early 1990s [1]. In these measurements, charge was deposited underneath one electrode of the device, using a red laser, and the collection was monitored. More recently, measurements have been made where charge has been deposited at various positions throughout the depth of a detector using an infrared laser [2]. Planar detectors before and after irradiation have been analysed using this method. Prior to the work reported here, only full thickness 3D devices had been studied using this technique [3]. This will be the first scan of a double-sided 3D detector.

Short, double-sided 3D, p-type strip detectors (produced at CNM Barcelona) have been used for this study [4]. A schematic of these detectors is displayed in figure 1. The strip detectors had a substrate thickness of $230 \pm 15$ micrometers and a strip pitch of 80 micrometers. The columns that formed the electrodes had a diameter of 10 micrometers, and were $170 \pm 10$ micrometers deep. The p-type columns were not fully etched since the strip detectors were placed at the edge of the wafer during fabrication where the Reactive Ion Etching process was not uniform. This resulted in columns with a depth of about 130 micrometers instead of the intended 170 micrometers. The columns were arranged as a square array with the same type columns at a pitch of 80 micrometers. The array of junction columns were displaced by half a pitch in both orthogonal directions to the ohmic columns, such that they lie at lay at the centre of the square by the ohmic columns. The n-type junction electrodes were connected together with 20 micrometer wide Aluminium metallisation to form the strips. Each readout strip was $4 \mathrm{~mm}$ long and consisted of 50 junction columns connected together. The Ohmic electrodes were connected together on the back-side of the device with a uniform Aluminium contact. These devices use a similar technology as to that used for the ATLAS IBL 3D pixel sensors [5]. The detectors were tested both prior to irradiation and after irradiating with reactor neutrons from the Jozef Stefan Institute reactor [6], to a dose of $5 \times 10^{15} 1 \mathrm{MeV}$ 


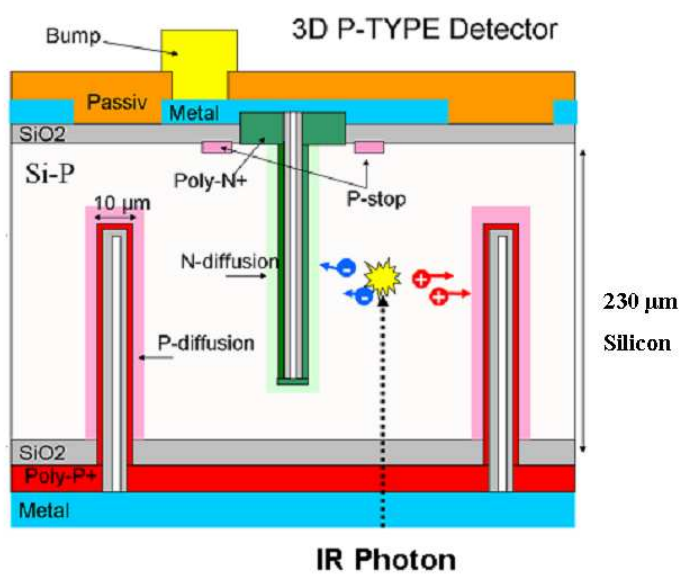

(a)

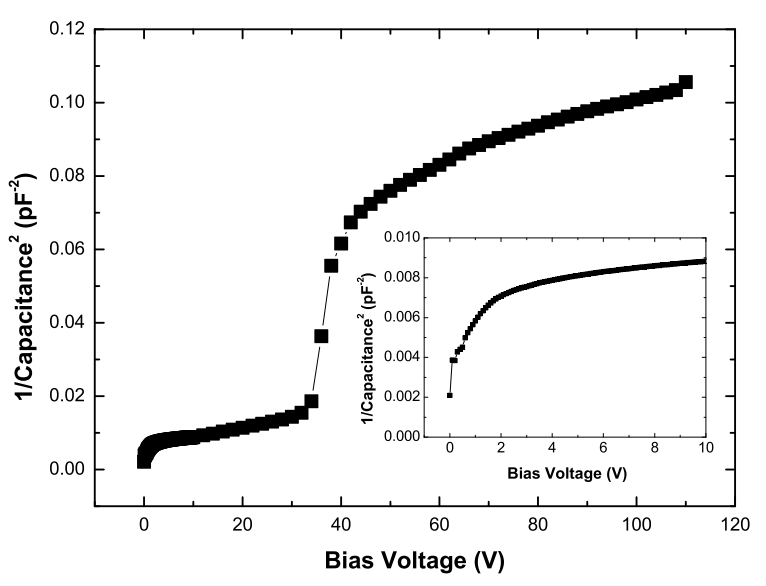

(b)

Figure 1. (a) Schematic of a p-type double-sided 3D detector and (b) related CV curve. The CV curve insert shows the inter-column region depletes at $3 \mathrm{~V}$ while the main plot shows the under-column region depletes at $40 \mathrm{~V}$ [7].

equivalent neutrons $/ \mathrm{cm}^{2}$. Studies were performed into the effect of varying bias voltage and also the effect of annealing on the irradiated sample.

An IR laser (1064 nm, pulse width of $0.1 \mathrm{~ns}$ ) was used to scan the devices with the spot focused on the centre of the bulk silicon. The spot had a diameter with a FWHM equal to 7 micrometers. The detector itself was mounted on a copper block with a Peltier element that allowed the temperature to be controlled from $-20^{\circ} \mathrm{C}$ to $60^{\circ} \mathrm{C}$. A Picosecond Pulse Labs Model 5531 bias-T was used to connect the high voltage power supply. This has a bandwidth of $1 \mathrm{MHz}$ up to $10 \mathrm{GHz}$. This then decoupled the readout electronics from the high voltage. Eleven strips adjacent to one another were bonded up, with the central strip connected to a wide band amplifier for readout. An oscilloscope then digitized and recorded the induced currents produced by the laser illumination. Scans with a step of 2.5 micrometers were performed over the surface of the device in both orthogonal directions, illuminating either the front surface or the cut edge (perpendicular to the strips). The data was recorded for the $10 \mathrm{~ns}$ before the laser pulse and the $40 \mathrm{~ns}$ after the laser pulse. The DAQ was triggered on the pulse from the laser driver, for each laser pulse with the final result for any illumination position taken as the average of many pulses. The laser pulses generated charge pairs along the path of the beam. The total signal is therefore an integration of the device's response throughout its depth. Illumination of non-active areas (metallisation or columns) resulted in reduced signals. The metallised areas will prevent the photons from penetrating into the silicon and producing any charge carriers. Any charge carriers produced in the electrode columns do not, or only partly, contribute to the signal. The baseline was corrected based on normalising the $10 \mathrm{~ns}$ prior to the pulse and extrapolating that to the rest of the signal. For a full description of the TCT technology and methods, refer to reference [8].

In order to reduce the diffraction of the light and hence reduce the beam width, for the edge illumination studies, the cut edge was polished prior to illumination. The laser beam illuminated the cut edge perpendicular to the aluminium strips on the top surface. The readout strip was several 
millimetres from the cut edge. In order for the laser light to reach the readout strip, around 40 inactive strip needed to be passed through. Where this was purely the silicon substrate, no degradation in the signal would occur. However, when the photons had to pass through multiple, inactive columns there was a consequential loss of signal due to reflections at the silicon, polysilicon and air boundaries within the columns. From an analysis with Fresnel's equations, this was estimated to lead to around a $4 \%$ loss of signal due to reflections. After 40 columns, only around $20 \%$ of the initial signal remained. The irradiation and edge polishing were completed at the Jozef Stefan Institute in Ljubljana [2]. The polishing was accomplished firstly by a rough grinding to get to the desired distance from the guard rings. This controls the absorption in the non-active part of the sensor. After that, a fine polishing was completed by using a diamond powder paste. The resulting surface was flat to the sub-micron level.

The TCT experiment was undertaken in an atmosphere of dry air, with the irradiated samples maintained at a temperature of $-20^{\circ} \mathrm{C}$. The non-irradiated samples were kept at room temperature with no temperature control, causing a greater leakage current than for the equivalent irradiated sample. The leakage current was recorded for each setup. Annealing was achieved in situ by warming to $60^{\circ} \mathrm{C}$ for intervals of $20,40,100,300$ and 600 minutes corresponding to room temperature annealing times of between 8 days and 200 days [9]. 300 minutes is equivalent to the amount of annealing expected for 7 years of operation in an LHC experiment, where (for example in ATLAS) it is estimated that the silicon sensors will be at $20^{\circ} \mathrm{C}$ for 30 days per year of operation.

\section{Results}

\subsection{CV and IV characteristics}

Figure 1(b) shows the capacitance-voltage characteristics of a non-irradiated short strip 3D detector. The curve demonstrates two distinct sections which relate to the two depletion regions: the intercolumn region depletes at $3 \mathrm{~V}$ while the main plot shows the under-column region depletes at $40 \mathrm{~V}$ [7]. The CV characteristics of a 3D device after irradiation are shown in [10].

The leakage current characteristics are shown for each step in figure 2. All the leakage currents after irradiation were measured with a detector temperature of $-20^{\circ} \mathrm{C}$. The device before irradiation was measured at $20^{\circ} \mathrm{C}$ and scaled to the equivalent at $-20^{\circ} \mathrm{C}$. The lateral full depletion voltage of the irradiated device is around $100 \mathrm{~V}$ [10], corresponding to the plateauing of the leakage current around that bias voltage. For bias voltages above this value, soft breakdown occurs as is often the case in irradiated detectors. Annealing decreases the leakage current of the device, as was expected. This is because the short periods of annealing provided beneficial annealing. This beneficial annealing reduced the current related damage rate, $\alpha$, by restructuring the lattice into a configuration that reduces the leakage current.

\subsection{Edge TCTs for non-irradiated sensors}

TCT measurements of the cut edge were performed only for non-irradiated devices and are therefore shown first. Waveforms were collected for laser pulses at a series of different positions on the cut edge and shown in figure 3 . The back-side, $\mathrm{p}^{+}$-type, ohmic columns extend downwards 


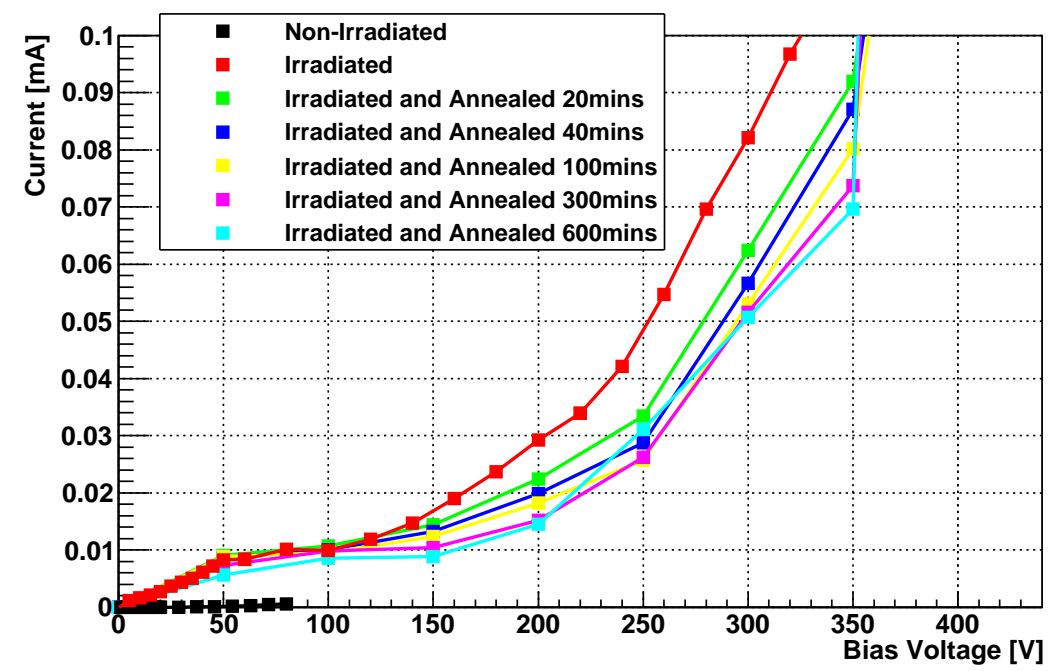

Figure 2. Leakage current against reverse bias voltage for the non-irradiated and irradiated sample and for each annealing step.

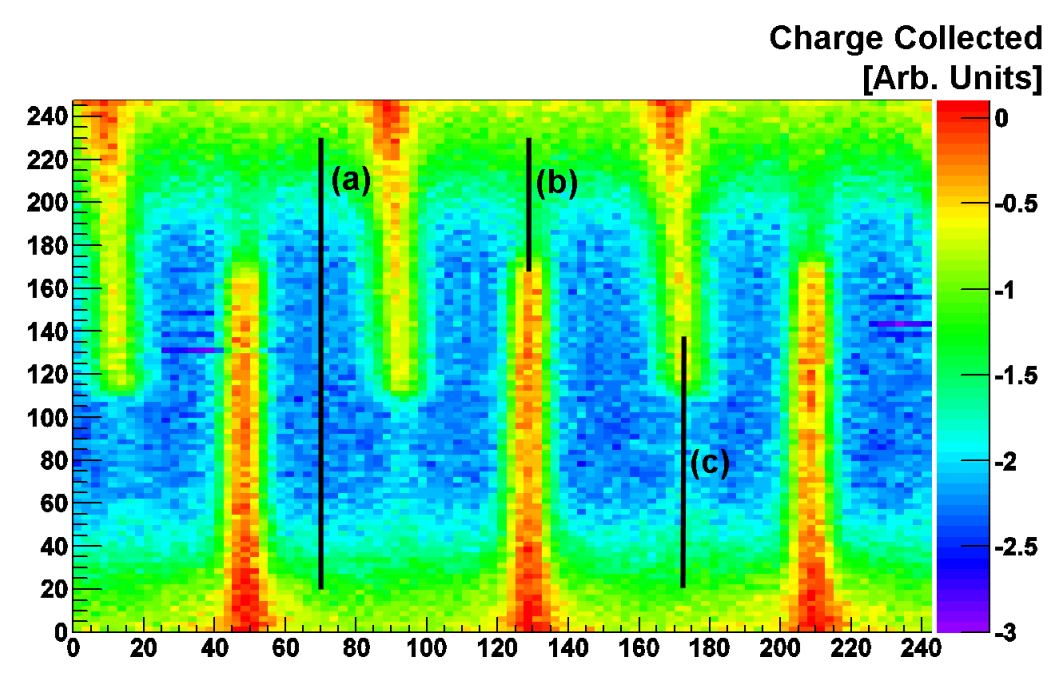

Figure 3. Charge collection map for non-irradiated device with edge illumination. Bias voltage was set to $20 \mathrm{~V}$. Lines (a), (b) and (c) correspond to the waveforms in figures 4(a), (b) and (c). Columns extending from the top of the image (at x-positions 10, 90 and $170 \mu \mathrm{m}$ ) are the p-type, ohmic columns, and the opposing columns (at x-positions 50,130 and $210 \mu \mathrm{m}$ ) are the n-type, junction columns. The areas of more negative signals have the greater charge collection. The $\mathrm{x}$ and $\mathrm{y}$ axes are distances in micrometers.

from the top of the figure and the front-side, $\mathrm{n}^{+}$-type, junction columns extend from the bottom. The charge collection plot was obtained for an integration time of $20 \mathrm{~ns}$ and a bias voltage of $20 \mathrm{~V}$. The collected charge was recorded as a negative signal. As the laser light penetrates deep into the device, charge is produced at every position along the laser beam. Therefore, the signal observed at the position of the columns is due to material in front of and behind the columns. Areas of high charge collection between areas of low charge collection are observed. The areas of low charge collection correspond to the positions of the columns. This agrees with the previous simulations 


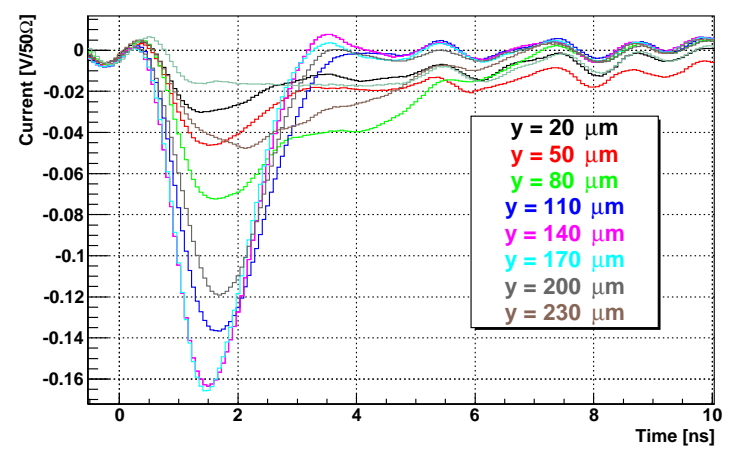

(a)

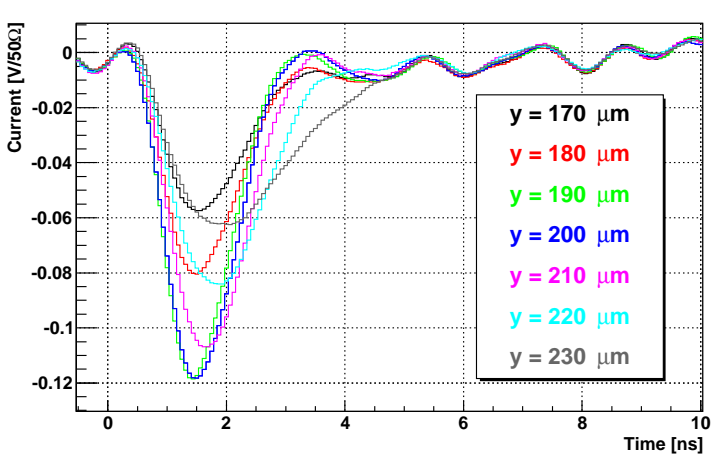

(b)

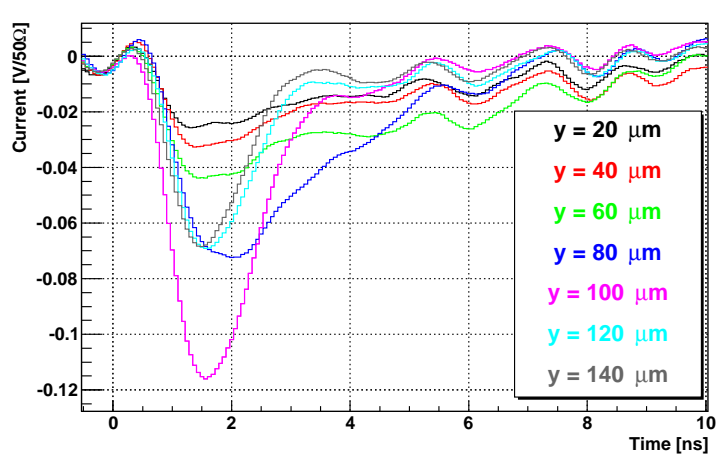

(c)

Figure 4. Waveforms for various positions of illumination for a bias voltage of $20 \mathrm{~V}$. An increase in the time required for the peak charge shown as the position moves towards the edge of the device.

completed [11]. The bias voltage of $20 \mathrm{~V}$ is greater than the lateral depletion of $3 \mathrm{~V}$, but less than the full depletion beneath the columns of $40 \mathrm{~V}$.

The waveforms from which the charge collection map was created are shown in figure 4 . From these, the major peak of the signal and the majority of the charge was collected within the first few nanoseconds but an integration time of $20 \mathrm{~ns}$ was required to fully collect the charge. For longer integration times, a reflection of the signal along the cable between the amplifier and the oscilloscope caused an erroneous result to be produced. Another aspect of the charge collection that can be analysed with the waveforms is the rate of charge collection. When considering illumination for positions between the columns, shown in figure 4(a), the earliest peaks and consequently the fastest signals occur in the centre of the device, shown for waveforms collected at 140 and $170 \mu \mathrm{m}$. This corresponds to the area of highest field strength [11]. In the region of high collection efficiency below the columns seen in figures 4(b) and 4(c)) the total charge collected is constant. However, the collection of charge takes a longer time as the position of illumination moves away from the column, as shown in the waveforms at $230 \mu \mathrm{m}$ in figure 4(b) and $80 \mu \mathrm{m}$ in figure 4(c). The columns of each type appear to have physical differences between them. The junction columns appear to penetrate to $170 \mu \mathrm{m}$ whereas the ohmic columns only appear to reach $135 \mu \mathrm{m}$.

The data collected for a scan between the two column types as a function of position from the back $(0 \mu \mathrm{m})$ to the front $(245 \mu \mathrm{m})$ side of the detectors were used to determine the charge collection 


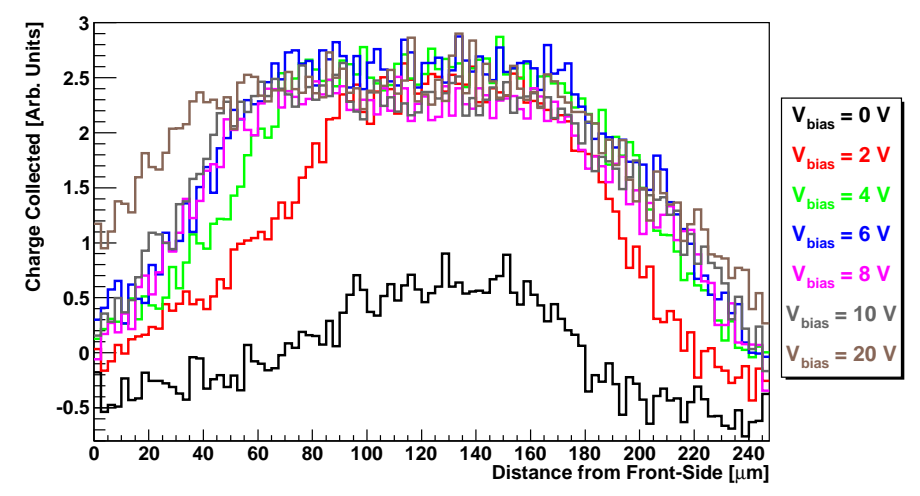

Figure 5. Charge collected as a function of distance at different detector biases. The distance is measured from $0 \mu \mathrm{m}$ at the front-side of the detector to $245 \mu \mathrm{m}$ at the back-side of the detector. The lateral illumination position was halfway between n-type and p-type columns.

profile at different bias voltages, as plotted in figure 5. The collected charge was measured by integrating the current in $20 \mathrm{~ns}$ after the laser pulse. At $0 \mathrm{~V}$ there is some charge collection due to the built in potential creating a small depletion region around the n-type columns. It can be seen that the charge collected in the inter-column region saturates at voltages above the lateral depletion voltage of $3 \mathrm{~V}$. At a bias voltage of $4 \mathrm{~V}$, just above full lateral depletion, full charge collection is obtained from $70 \mu \mathrm{m}$ to $180 \mu \mathrm{m}$. At higher bias voltages the charge collected in this region remains constant, while the region extends in both directions, such that at $20 \mathrm{~V}$ full charge collection is obtained from $40 \mu \mathrm{m}$ to $190 \mu \mathrm{m}$. $50 \%$ of full charge collection is obtained from $15 \mu \mathrm{m}$ to $215 \mu \mathrm{m}$ at this bias voltage.

Maps of the charge collected over the entire edge of the device were obtained for increasing bias voltages, and shown in figure 6 . It can be seen that the charge collected increases with the bias voltage. The inter-column region is depleted at biases of $4 \mathrm{~V}$ and above, with the region beneath the columns depleting at greater biases. A low field region with reduced charge collection can also be seen where the columns connect with the front and back surfaces of the detector. This low-field region reduces in size as the bias voltage increases.

From the charge collection maps the widths of the $\mathrm{p}$ and n-type columns were calculated and then compared to previous experimental measurements [12]. Gaussian curves were fitted to the charge collection at the mid-depth of the device $(130 \mu \mathrm{m})$ at each bias point, with the FWHM taken as the width of the column. This is displayed in figure 7. Bias voltages below the depletion voltage $(3 \mathrm{~V})$ were not included in the final measurement as the edges of the columns were less well defined. This gave an average value of $7.30 \pm 0.03 \mu \mathrm{m}$ for the p-type column and $9.18 \pm 0.03 \mu \mathrm{m}$ for the n-type column. This agrees with the previous analysis [12]. The measurements made during the fabrication process [4] suggest that the columns should be $10 \mu \mathrm{m}$ wide in the middle of the column and taper to 5 or $6 \mu \mathrm{m}$ wide at the column's tip. The discrepancy between the expected diameter and the measured diameters is a result of the detectors being on the edge of the original wafers where the column formation was not as uniform.

The rise time of the waveforms is the time taken for the amount of charge collected to increase from $10 \%$ to $90 \%$ of the maximum [2]. For our system this varied from $0.5 \mathrm{~ns}$ to $0.75 \mathrm{~ns}$. By fitting 


\section{Charge Collected}

[Arb. Units]
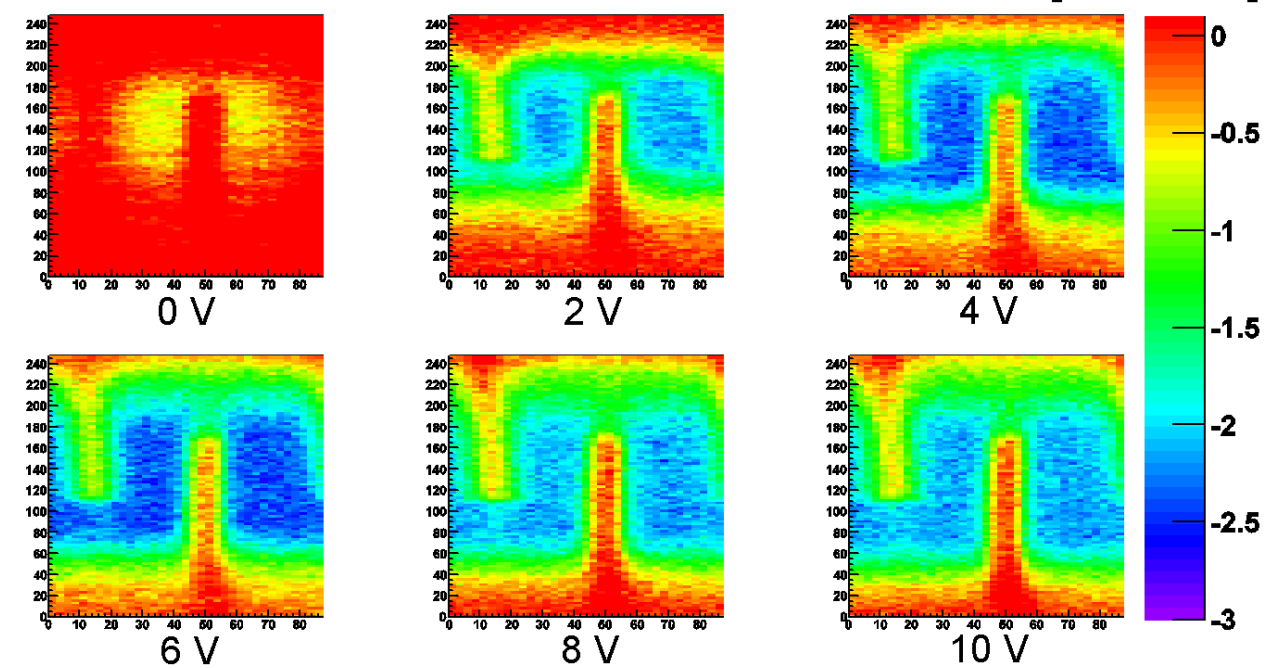

Figure 6. Charge collection maps with edge illumination, with bias voltages increasing from $0 \mathrm{~V}$ (top left) to $10 \mathrm{~V}$ (bottom right) in steps of $2 \mathrm{~V}$. For the charge collection at $20 \mathrm{~V}$, refer to figure 3.

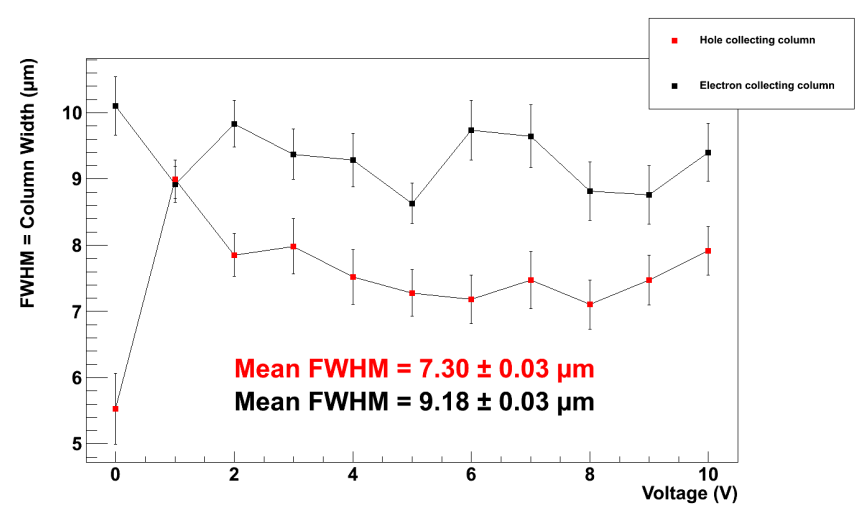

Figure 7. FWHM values for increasing bias voltages. Average calculated for bias voltages above the depletion voltage of $3 \mathrm{~V}$.

a second order polynomial to this range, any trapping effects can be ignored and the velocity profile of the charge carriers can be determined from the current. It is not possible with this technique to determine the contribution towards the velocity derived from either the electrons or holes independently. This method assumes that all of the charge carriers were generated in the same field. This is certainly true in the region of overlap in the columns.

Velocity profiles were produced for three different illumination regions, shown in figure 8. The three regions were for a line scan under the front-side column; beneath the back-side column and at the mid-point between the front and back-side columns (shown in figure 3 as lines (a), (b) and (c)), with the beam moving from the front-side to the back-side.

Firstly, every position shows an increase in velocity as the bias voltage increases due to the uniform increase in the electric field with bias voltage. This is in contrast to the charge collection, 


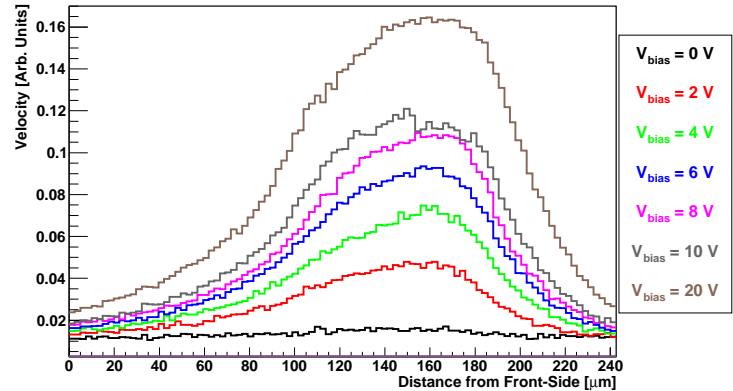

(a)

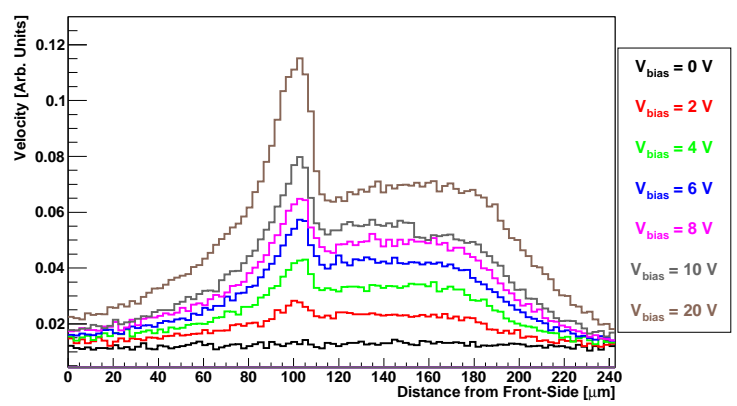

(c)

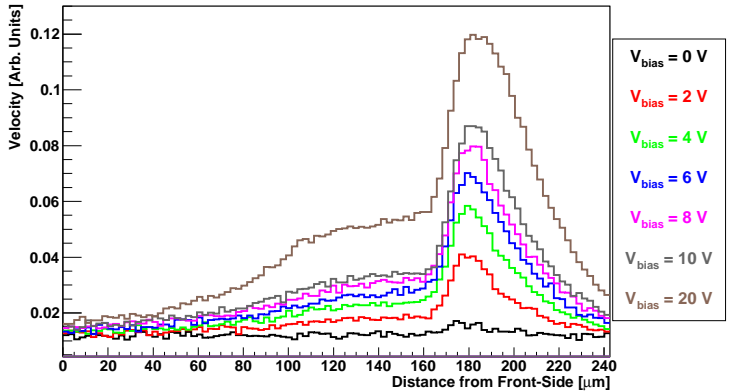

(b)

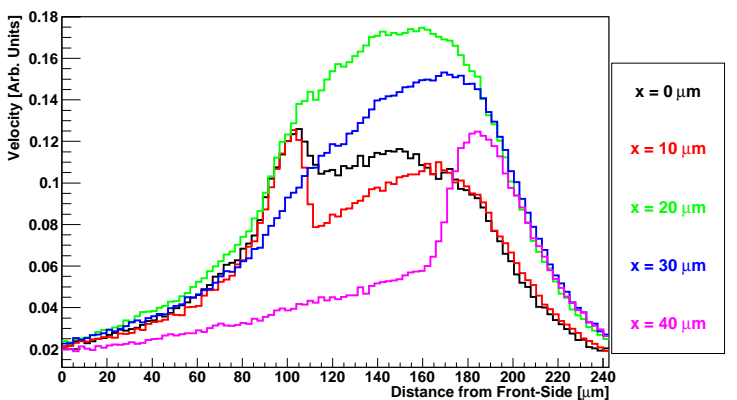

(d)

Figure 8. Velocity profiles of a non-irradiated sensor with various bias voltages and edge illumination. (a) corresponds to a line of illumination positions halfway between n-type and p-type columns, (b) and (c) correspond to positions collinear with the p-type and n-type columns respectively. (d) shows a series of profiles with different starting x positions and a bias voltage of $20 \mathrm{~V}$.

shown previously in figure 5. Whereas the full charge is collected in $20 \mathrm{~ns}$ at $4 \mathrm{~V}$, the carrier velocity and therefore electric field continues to increase with bias voltage. The maximum velocities, shown in figure 9 , do not increase linearly but are suppressed by the saturation of the field in the central region. Also, if we compare the charge collected and the carrier velocity at $40 \mu \mathrm{m}$ from the frontside of the device, for a bias voltage of $20 \mathrm{~V}$, we see that although there is only a small carrier velocity relative to the middle of the device we get close to full charge collection due to the short distance traveled by the carriers. Secondly, the detector shows greater charge collection towards the surface at higher bias voltages because the low-field region near the surfaces is still depleting. At $2 \mathrm{~V}$ only around $100 \mu \mathrm{m}$ of the sensor shows full charge collection, rising to the full $230 \mu \mathrm{m}$ at $20 \mathrm{~V}$.

The velocity given for an illumination position within a column is non-zero because of the active silicon before and after the column that the beam also passes through, shown in figure 8(c) from $0 \mu \mathrm{m}$ to $160 \mu \mathrm{m}$ and in figure $8(\mathrm{~d})$ from $110 \mu \mathrm{m}$ to $230 \mu \mathrm{m}$. There is a peaked velocity close to the column tips for both the $\mathrm{n}$ and $\mathrm{p}$-type columns due to the high electric field in these regions.

The peak velocity below the columns (at $100 \mu \mathrm{m}$ and $200 \mu \mathrm{m}$ ) is equivalent to the velocity at the same position in the inter-column region because the signal is dominated by the charge carriers moving in the active silicon away from the column and the high fields at the column tips are obscured. This is demonstrated in figure 8(d) by a series of five different illumination regions 


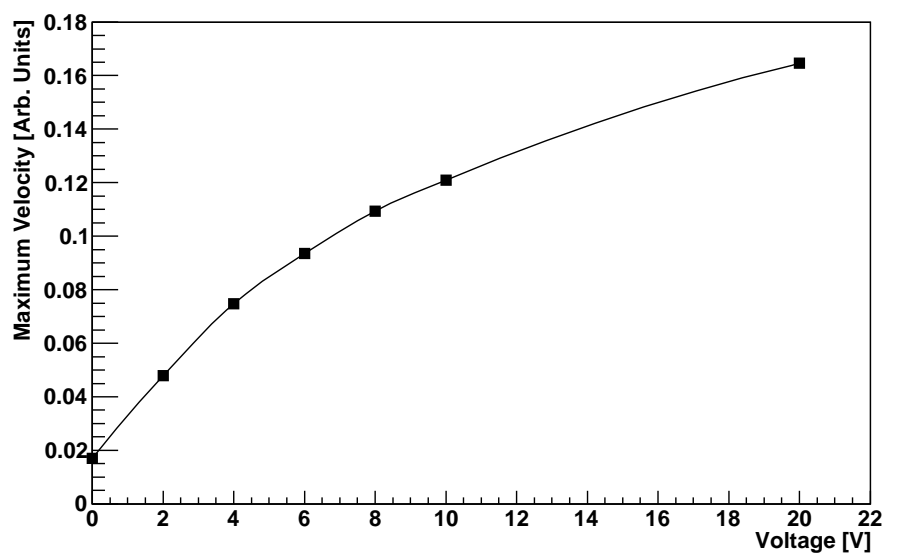

Figure 9. Maximum velocities of charge carriers generated $20 \mu \mathrm{m}$ from either ohmic or junction columns at a variety of bias voltages.

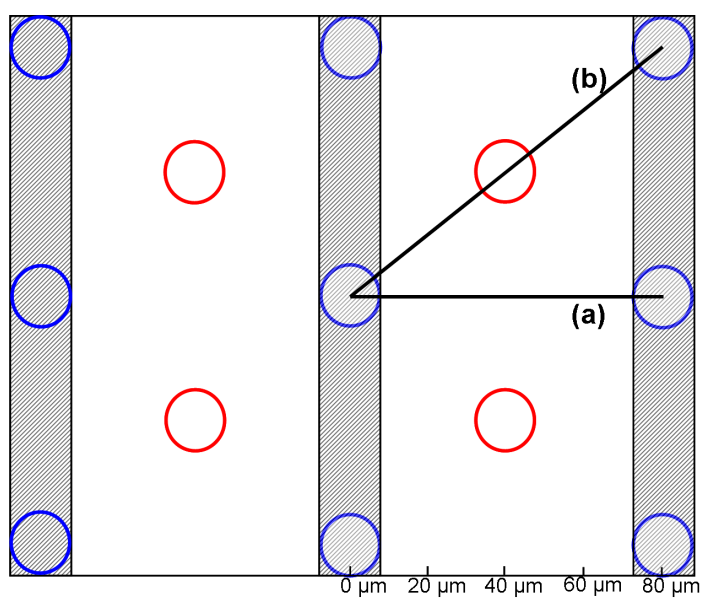

(a)

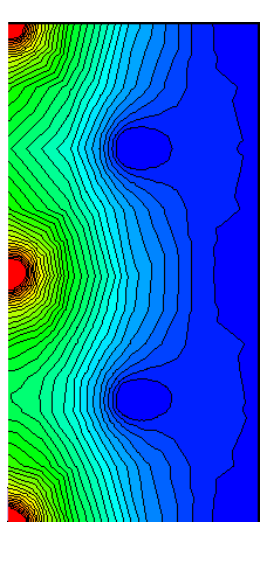

(b)

Figure 10. (a) shows a schematic of the top surface that was scanned. Lines (a) and (b) correspond to the waveforms in figures 11(a) and (b). Positions of n-type columns (blue circles), p-type columns (red circles) and strips (hatched areas) have been indicated. (b) is a simulation of the weighting field for the device, with contour lines showing the weighting potential. Each contour line represents a change of 0.03 in weighting potential. The cross-section is halfway down the device.

with a bias voltage of $20 \mathrm{~V}$. The initial positions for each scan began at $0 \mu \mathrm{m}$ at line (c) and then moved across in steps of $10 \mu \mathrm{m}$ to the adjacent column.

\subsection{Spatially resolved top surface TCTs for non-irradiated sensors}

TCT measurements were also performed for illumination normal to the top surface of the nonirradiated sensor. Again, waveforms were collected for laser pulses at a series of positions across the surface. A schematic of the top surface being scanned is shown in figure 10(a). The current induced on a collecting electrode is related to the charge produced and the weighting field by the Shockley-Ramo Theorem [13]. A simulation of the weighting field for the strip devices we tested is shown in figure $10(b)$. 


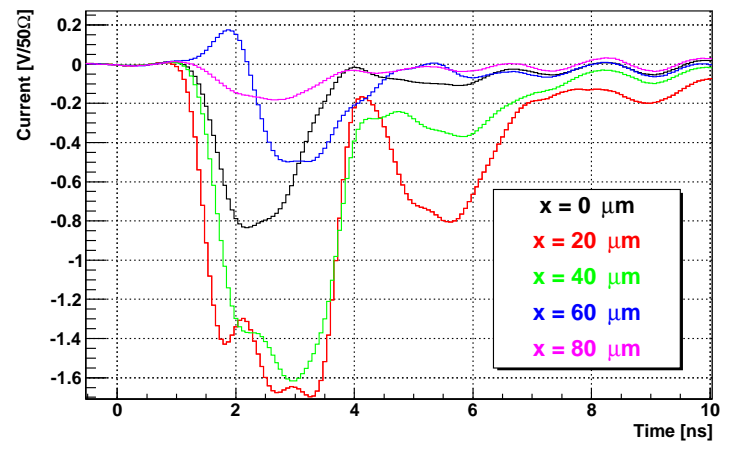

(a)

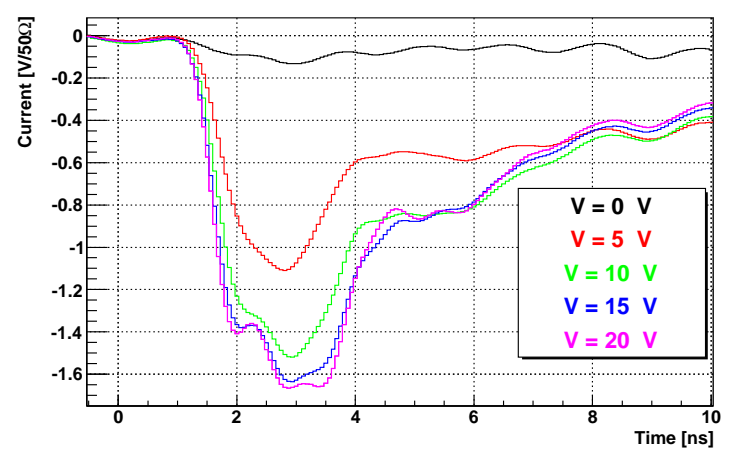

(c)

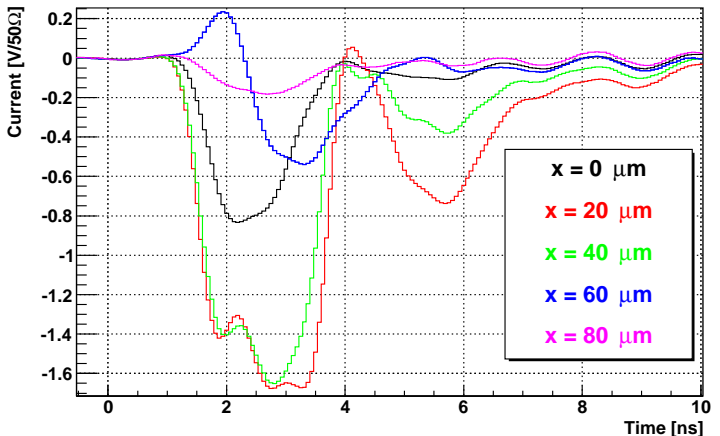

(b)

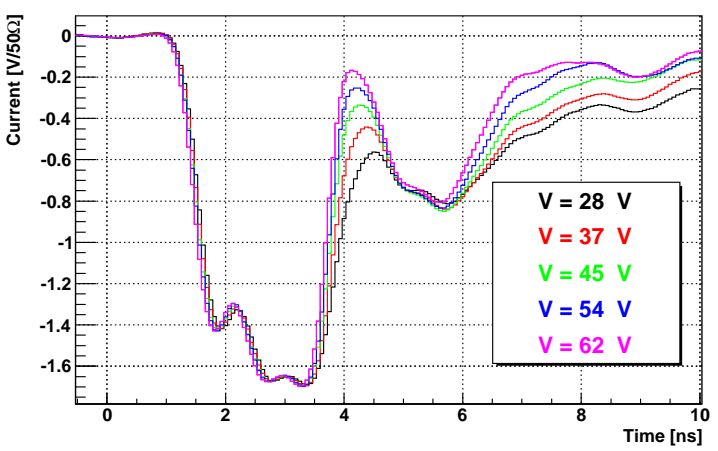

(d)

Figure 11. (a) and (b) show waveforms from various positions of illumination over the top surface of a nonirradiated sensor with a bias voltage of $62 \mathrm{~V}$. The position of illumination is given by the lines in figure 10(a). (c) and (d) show the effect of varying the bias voltage on waveforms at a position of illumination $20 \mu \mathrm{m}$ from the central strip on line (b).

According to Ramo's Theorem, the signal induced on the electrodes of the detector are due to the movement of the charge carriers inside the device. This is given as a dot product of the velocity vector of the charge carriers and of the weighting field. Therefore carriers that experience a large change in weighting field during their movement through the device induce more signal than those that travel through regions of small weighting field change. This effect is exploited in the characterisation of planar devices with front and back-side alpha or short wavelength illumination to obtain the charge collected from individual charge carrier types. For a 3D detector, the situation is slightly different. Illumination close to the $n+$ junction column will result in the holes produced inducing the majority of the signal; while illumination close to the $\mathrm{p}+$ ohmic column will result in a signal dominated by electron movement. Illumination mid-point between the junction and ohmic columns gives rise to a signal due approximately equally to both electrons and holes.

Figure 11(a) shows a series of waveforms starting at the electron collecting column and moving in steps of $10 \mu \mathrm{m}$ perpendicular to the strip. Figure 11(b) shows another series of waveforms, this time progressing from the electron collecting column at $45^{\circ}$ to the strip. For both data sets, the waveform at $0 \mu \mathrm{m}$ show a reduced response. This is because the majority of the laser pulse is reflected by the metallised strip with the rest being caused by the extremities of the beam spot. At $20 \mu \mathrm{m}$ from the electron collecting column, the waveforms display two major peaks. There are 
several interesting effects inside the device that can be seen from the current pulses obtained and shown in figure 11.

Figure 11(a) and 11(b) show that for over depletion, $62 \mathrm{~V}$ bias, the signal duration is of the order of $3 \mathrm{~ns}$ with a second peak, of $2 \mathrm{~ns}$ duration, occurring a nanosecond after the first. The origin of the second peak is at present not fully understood, but is suspected to be due to the column non-overlap region of the device. The structure of the main first peak depends on illumination position and bias voltage. Figure 11(a) shows the waveforms for different illumination positions along line a shown in figure 10(a). The signal for illumination at at $\mathrm{x}=0 \mu \mathrm{m}$, close to the junction column, shows a signal that has a falling magnitude with time. This is due to the velocity of the signal charge carriers falling with time. This signal is dominated by the movement of holes away from the junction electrode towards the ohmic electrode. The signal at $x=40 \mu \mathrm{m}$, corresponding to a position between two ohmic columns and $40 \mu \mathrm{m}$ away from the strip, is dominated by the movement of electrons created between two ohmic contacts drifting to the junction electrode. The movement of holes to the ohmic contact will not induce significant signal as the change in weighting field over this region is small, as can be seen in figure 10(b). The waveform shows an increasing signal with time which is as expected for the electrons moving into a region with a greater electric field, and therefore moving with increasing speed with time. The signal produced at $x=20 \mu \mathrm{m}$ is due to both the movement of electrons and holes in approximately equal measure. The rise time of the signal at $\mathrm{x}=20 \mu \mathrm{m}$ is faster than that at $\mathrm{x}=40 \mu \mathrm{m}$ due to the higher electric field at this point and therefore higher carrier velocities. The hole signal will take a little longer than the electron signal due to their lower mobility. This is clear in the wider signal compared to that from $x=40 \mu \mathrm{m}$, which is in fact due to electrons moving over twice the distance as for the $\mathrm{x}=20 \mu \mathrm{m}$ signal.

The $\mathrm{x}=20 \mu \mathrm{m}$ signal exhibits three bumps. The first reduction in current (falling side of the first bump) is due to the movement of holes to a lower weighting field. The second bump is due to the electron signal dominating. The electrons move to the higher weighting field nearest to the electrode and therefore give rise to a signal with rising magnitude with time, as observed. The falling edge of this bump is due to the collection of electrons. The third bump is due to the movement of holes and is due to the fact that the hole velocity increases slightly as it approaches the ohmic columns.

The waveforms shown in figure 11(b) are very similar to those shown in 11(a). These are due to illumination at positions along line (b) in figure 10(a) which joins a junction column to an ohmic column. The significant differences in the waveforms are that the rise time of the signals produced at $\mathrm{x}=20 \mu \mathrm{m}$ and $\mathrm{x}=40 \mu \mathrm{m}$ are very similar, unlike in the waveforms of figure 11(a). This is due to the fact that along this line the electric fields in the device are higher than at the point between two ohmic contacts (figure 11(a) point $\mathrm{x}=40 \mu \mathrm{m}$ ) and therefore there is little difference in the velocities of the charge carriers for these two illumination positions.

At $60 \mu \mathrm{m}$, a bipolar signal is observed, due to the electrons being collected by the adjacent columns and inducing a signal of opposite polarity. $60 \mu \mathrm{m}$ is outside of the unit cell formed by the p-type columns at the for corners and the strip running through the centre. Electrons generated at this position will travel to the strip neighbouring the collecting strip. The bipolar peak results from the electrons having no effect on the collecting strip and thus integrating to zero. The holes generated here move to the same column as at a point any other distance along the line, however the hole signal is suppressed in this region because of the low weighting field. 


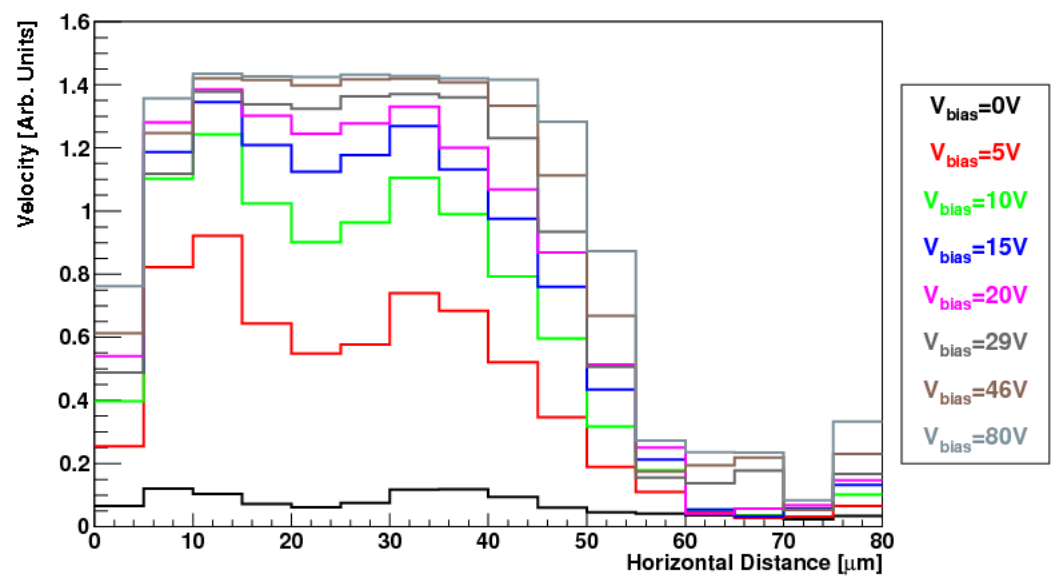

Figure 12. Velocity profiles of a non-irradiated sensor with various bias voltages for a line segment passing through several columns. N-type columns are at $0 \mu \mathrm{m}$ and $80 \mu \mathrm{m}$ with a p-type column at $40 \mu \mathrm{m}$. The position of illumination is given by the line (b) in figure 10 (a).

Figures 11(c) and 11(d) show the waveforms produced with increasing bias voltage for the illumination point of $x=20 \mu \mathrm{m}$ along line (b) of figure 10(a). The waveform generated with a bias voltage of $5 \mathrm{~V}$ shows only an increasing current signal with time. This is due to the fact that the signal is dominate by electron movement towards the junction column because, while the device is fully depleted laterally, the magnitude of the electric field close to the ohmic columns is low and the signal from the holes drifting into this region is small. As the bias voltage increases the magnitude of the electric field inside the device increases and the waveforms exhibit distinct peaks forming, at approximately $15 \mathrm{~V}$, due to the increase in the contribution of the hole signal, due to increasing hole velocity in the device in the region around the ohmic contact.

The rise time and magnitude of the prompt signal saturates at about $20 \mathrm{~V}$. The rise time is dominated by the movement of the electrons in the high field region around the junction electrode, and this saturates as the velocity of the electrons in this region of the device saturates. The fall time of this peak continues to become faster with increasing bias voltage, as this is dominated by the collection of holes in the device moving in the region around the ohmic contact where the electric field is lower than around the junction contact. The second displaced peak becomes separated from the first prompt peak with increasing bias voltage, due to a faster rise time and the fast fall time of the prompt peak. The second peak also has a faster fall time with bias voltage. However, the position of the peak of the signal does not change.

Using the same method as before, the velocity profile for the section between two opposite columns can be extracted and is presented in figure 12. These are taken along the line (b) in figure 10(a). Between $0 \mu \mathrm{m}$ and $5 \mu \mathrm{m}$ there is a small signal as a result of the non point source illumination. At $5 \mathrm{~V}$, there is significant signal across the whole detector as expected from the lateral depletion voltage of $3 \mathrm{~V}$. The combined electron and hole velocity is greatest in the $10 \mu \mathrm{m}$ adjacent to the columns implying high electric field regions exist around the columns. The velocity then decreases in the region between the columns, because while there is full lateral depletion at $5 \mathrm{~V}$ there is still significant non-uniformity within the field of the device. As the voltage is increased, the velocity in all regions increases until saturation is reached between $29 \mathrm{~V}$ and $46 \mathrm{~V}$. 

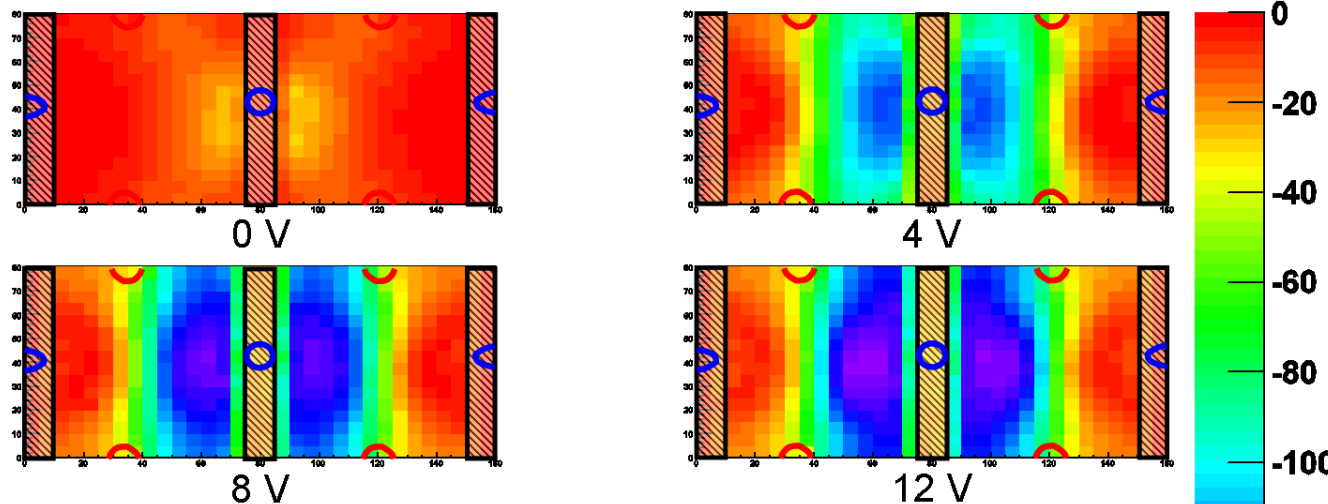

$8 \mathrm{~V}$
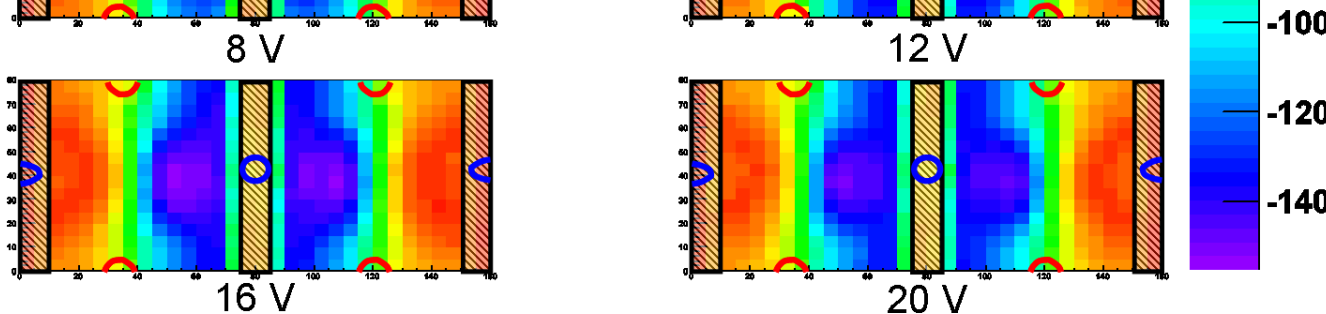

Figure 13. Charge Collection maps with top illumination for a non-irradiated sensor. Bias voltage increases from $0 \mathrm{~V}$ (top left) to $15 \mathrm{~V}$ (bottom right) in steps of $3 \mathrm{~V}$. Positions of n-type columns (blue circles), p-type columns (red circles) and strips (hatched area) have been indicated.

The charge collection maps, presented in figure 13, show the integrated charge collected in the $20 \mathrm{~ns}$ after the pulse. At $0 \mathrm{~V}$, charge is only collected around the n-type columns and in areas directly between the $\mathrm{n}$ and $\mathrm{p}$-type columns where the electric field is non-zero because of the builtin potential. There is little charge collected between columns of the same type because of the low electric field. As the voltage is increased, firstly the region around the n-type column shows full charge collection with the difference between the area around the n-type column and the rest of the active area disappearing as the voltage is increased to $15 \mathrm{~V}$. Even at $15 \mathrm{~V}$, the charge collection increases towards the collecting columns.

The area around the p-type column show a low level of charge collection for biases up to $15 \mathrm{~V}$. This is partially due to the column itself being non-collecting and also because the beam spot has a finite size. Although the centre of the beam may be outside of the column, part of the signal may be lost. There is still non-uniform charge collection in the low weighting field region between the adjacent p-type columns. The proportion of charge collected falls to 50\% at the boundary of the unit cell. Any charge deposited at this location has a chance of being shared with and collected by the neighbouring cell.

\subsection{Surface TCTs for irradiated sensors}

A sensor was irradiated to $5 \times 10^{15} 1 \mathrm{MeV}$ equivalent neutrons $/ \mathrm{cm}^{2}$ at the Jozef Stefan Institute in Ljubljana. The irradiated devices were kept at $-20^{\circ} \mathrm{C}$ to stabilise the leakage current and prevent unintentional annealing. During the initial mounting, the detector was at a slight angle to the orthogonal. This was corrected in later scans. As before, waveforms were collected for the same 


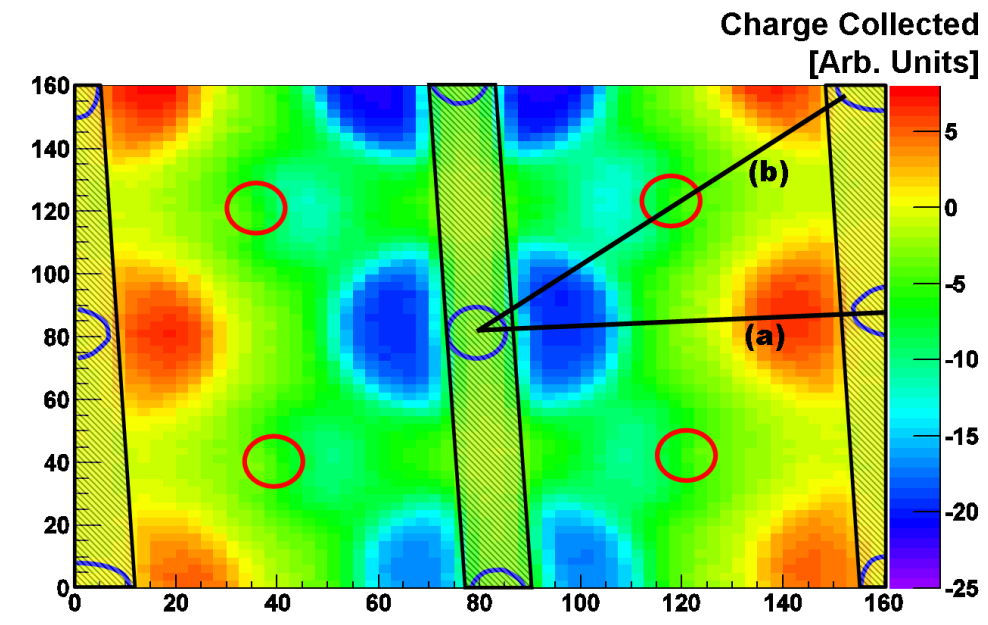

Figure 14. Charge collection map for the irradiated device with top surface illumination. Bias voltage was set to $70 \mathrm{~V}$. Lines (a) and (b) correspond to the waveforms in figures 15(a) and (b). Positions of n-type columns (blue), p-type columns (red) and strips (hatched area) have been indicated.

region across the surface and are shown in figures 14 and 15 . At $100 \mathrm{~V}$ there is very little difference between the waveforms generated perpendicular to the strip and those at $45^{\circ}$. The signal is much faster than for the non-irradiated case. This is due to the fact that the bias voltage applied is higher and the electric field is consequently greater. The trapping effects inside the device also reduce the duration of the signal. The signal at $100 \mathrm{~V}$ shows no obvious structure which implies that the contribution from the holes is small. The magnitude of the signals generated along the line between the junction and ohmic columns, shown in figure 15(b), falls uniformly with distance from the junction column as the electric field magnitude falls with distance from the junction column, even though the device has a bias of $100 \mathrm{~V}$ applied. The small signal at $\mathrm{x}=0 \mu \mathrm{m}$ is due to some of the signal being reflected by the metal strip.

With a higher bias voltage of $300 \mathrm{~V}$ the signal magnitude remains constant throughout the device (again the low signal at $x=0 \mu \mathrm{m}$ is due to reflections at the strip and at $x=40 \mu \mathrm{m}$ is due the laser falling on the non-sensitive hole). Therefore the device is fully active at this bias voltage. A second bump in the signal is now visible, but not a third, as in the non-irradiated case for illumination close to the ohmic contact. This would tend to suggest that the signal is still dominated by electrons even at these high bias voltages.

Velocity profiles from this sensor were extracted with the same method as before and are presented in figure 16. As with the non-irradiated sample, the velocity of the charge carriers are greatest when the pulse is adjacent to the n-type columns. Unlike the non-irradiated sensor however, the combined velocity is greatly suppressed around the hole collecting, p-type column, for bias voltage up to $80 \mathrm{~V}$. There is no evidence of a double field structure as expected for an irradiated device. The device is fully active, along this cross-section, at a low bias voltage ( $40 \mathrm{~V})$ but due to the low velocity at this bias point trapping will degrade the collected charge significantly.

The velocity throughout the device tends to increase with increasing bias voltage up to $200 \mathrm{~V}$. At $200 \mathrm{~V}$ the velocity has a flat profile between the columns. This would only be the case if the velocity had become saturated at these high bias values. 


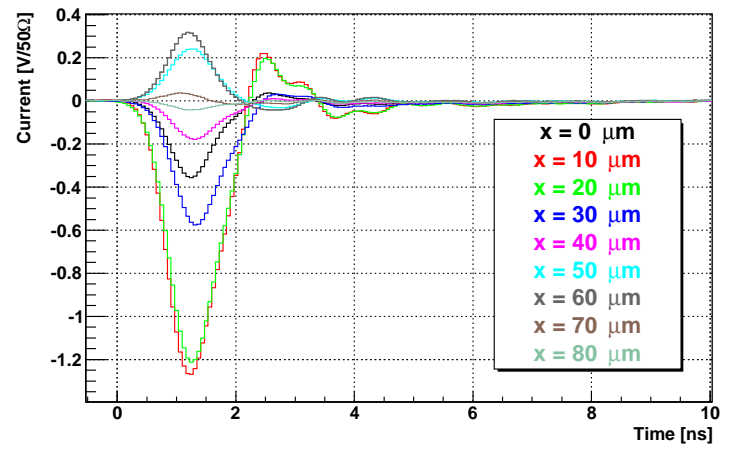

(a)

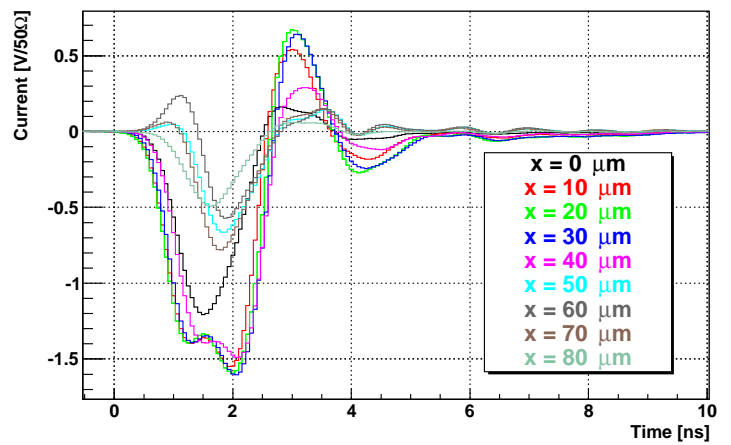

(c)

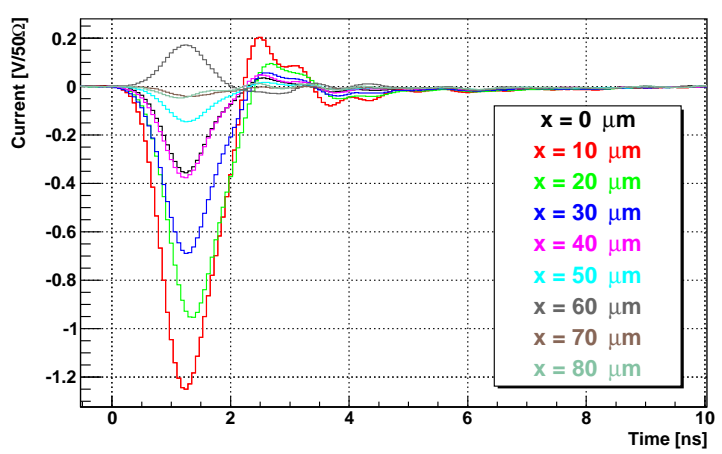

(b)

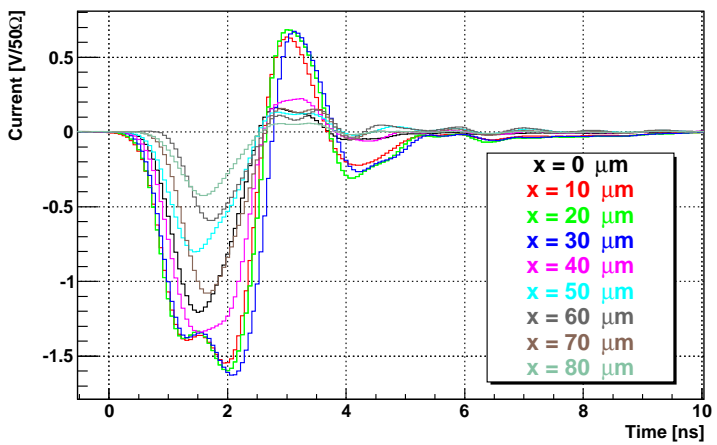

(d)

Figure 15. Waveforms from various positions of illumination over the top surface of an irradiated sensor moving from the central backside column to an adjacent one either perpendicularly in (a) and (c), or at $45^{\circ}$ in (b) and (d). Bias voltages shown were $100 \mathrm{~V}$ in (a) and (b) or $300 \mathrm{~V}$ in (c) and (d).

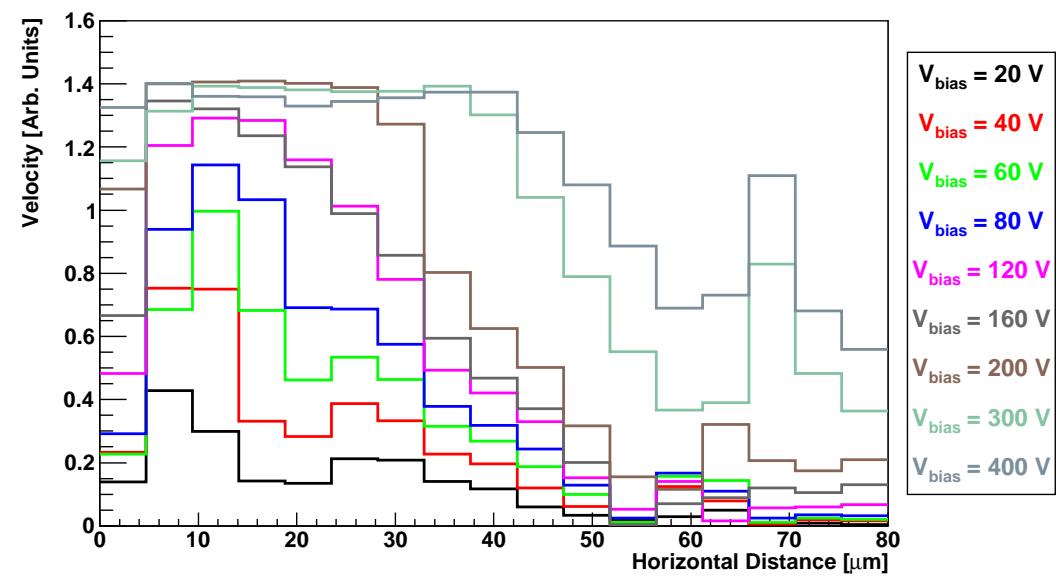

Figure 16. Velocity profiles of an irradiated sensor with various bias voltages for a line segment passing through several columns. N-type columns are at $0 \mu \mathrm{m}$ and $80 \mu \mathrm{m}$ with a p-type column at $40 \mu \mathrm{m}$.

A similar effect can be seen when considering the collected charge displayed in figure 17. Below the full depletion voltage, the majority of the charge is collected in the high field regions around the columns where the velocity is greatest. At higher voltages, a greater proportion of the 

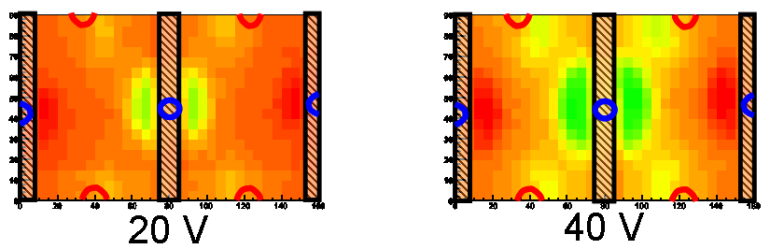

Charge Collected [Arb. Units]
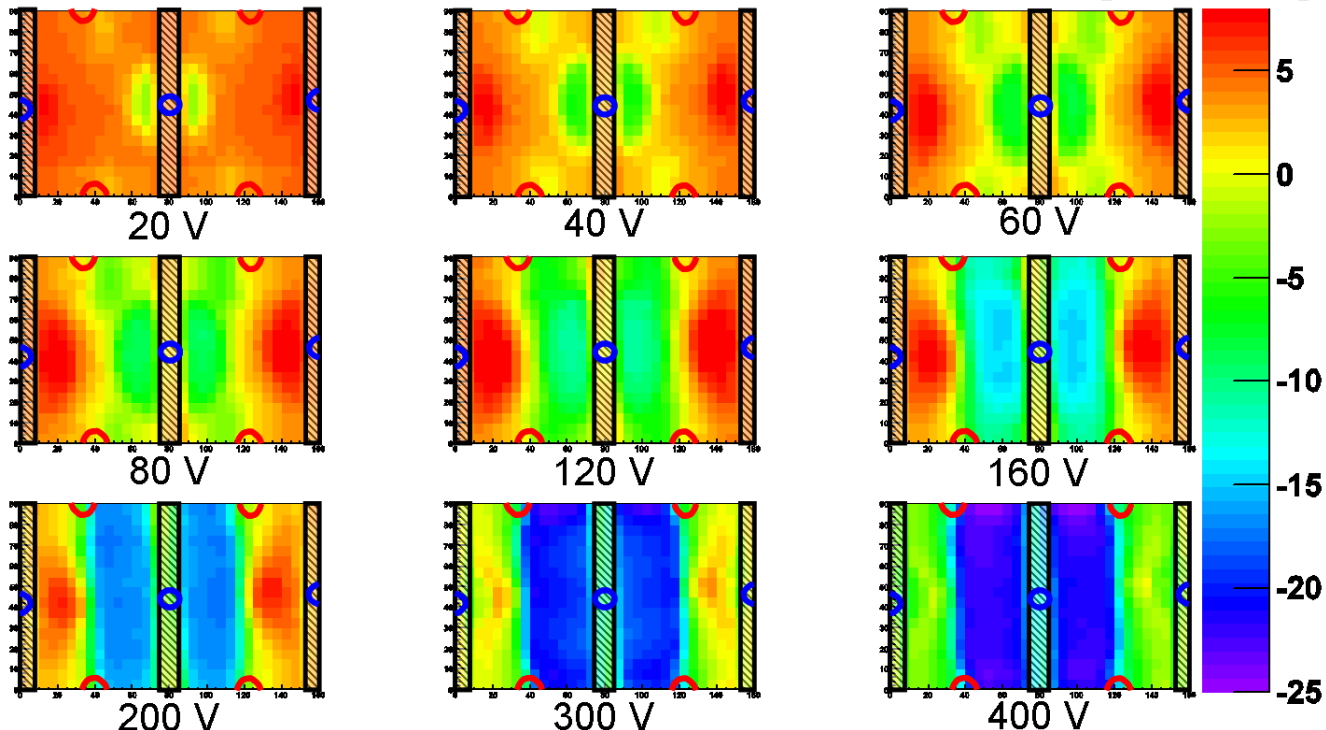

Figure 17. Charge Collection maps with top illumination for an irradiated sensor. The bias voltages shown are $20 \mathrm{~V}, 40 \mathrm{~V}, 60 \mathrm{~V}, 80 \mathrm{~V}, 120 \mathrm{~V}, 160 \mathrm{~V}, 200 \mathrm{~V}, 300 \mathrm{~V}$ and $400 \mathrm{~V}$. Positions of n-type columns (blue), p-type columns (red) and strips (hatched area) have been indicated.

charge is collected from lower field regions. The negative signals around the columns away from the central strip are due to the full charge not being collected because of ballistic deficit. Charge multiplication effects become apparent in the high field regions above $200 \mathrm{~V}$. Additional acceptors appearing during long term annealing enhance the field and the multiplication then becomes more obvious.

\subsection{Surface TCTs for irradiated sensors post-annealing}

The effects of annealing the samples upon the signal collected were studied using the same techniques described in section 2.3. The previous irradiated sample was annealed at $60^{\circ} \mathrm{C}$ for cumulative times of 20 minutes, 40 minutes, 100 minutes, 300 minutes and 600 minutes. Annealing for 600 minutes at $60^{\circ} \mathrm{C}$ (equivalent to the amount of annealing expected for 11 years of operation in an LHC experiment) led to the inter-strip resistance decreasing to a level where the strips were shorted together at bias voltages above $100 \mathrm{~V}$. For this reason, only the annealing steps of 0,20 , 40,100 and 300 minutes at $60^{\circ} \mathrm{C}$ were considered.

Looking at the charge collected in figure 18 and the waveforms generated after 20 minutes of annealing that are shown in figure 19, a similar pattern to the pre-annealing irradiated sample can be observed. The same peaks and sub-peaks are very similar. For a bias voltage of $100 \mathrm{~V}$ the signal collected for illumination points closer to the ohmic contact $(\mathrm{x}=30 \mu \mathrm{m})$ are now very similar to those collected for illumination close to the junction contact. This implies that the device has a high electric field throughout. At $400 \mathrm{~V}$ bias the waveforms are very similar to those before annealing.

Similarly to the non-irradiated device, it is possible to sum the charge generated between the columns as if a MIP traversed the region. This is presented in figure 21. At bias voltages between 


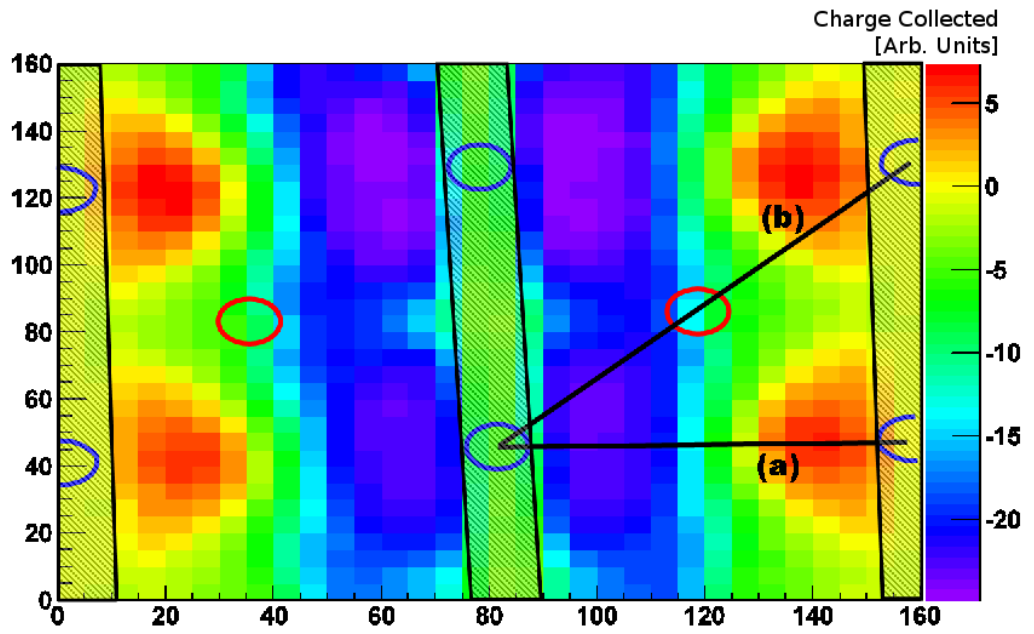

Figure 18. Charge collection map for the irradiated device after annealing for 20 minutes at $60^{\circ} \mathrm{C}$ with top surface illumination. The bias voltage was set to $100 \mathrm{~V}$. Lines (a) and (b) correspond to the waveforms in figures 19(a) and (b). Positions of n-type columns (blue), p-type columns (red) and strips (hatched area) have been indicated.

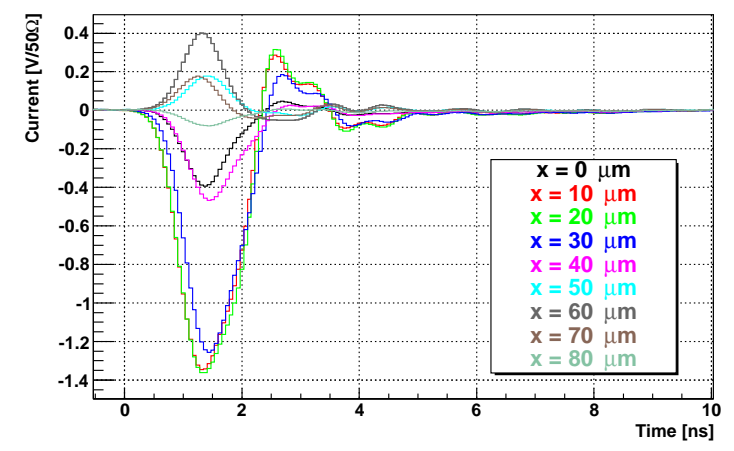

(a)

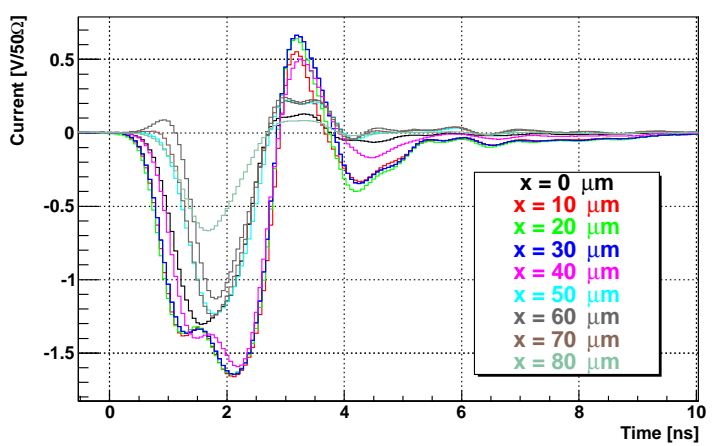

(c)

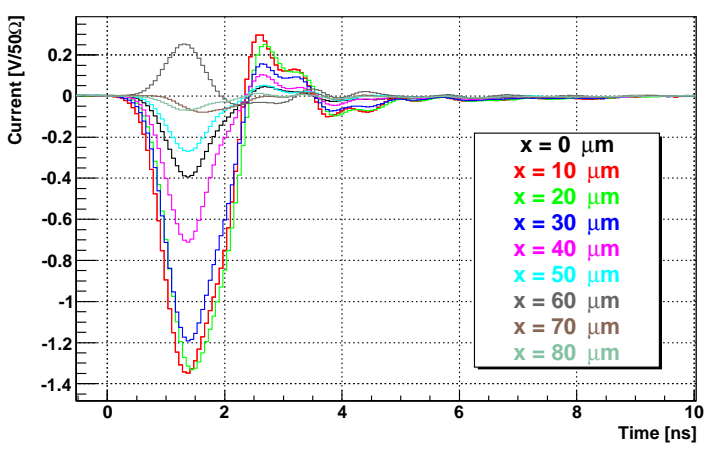

(b)

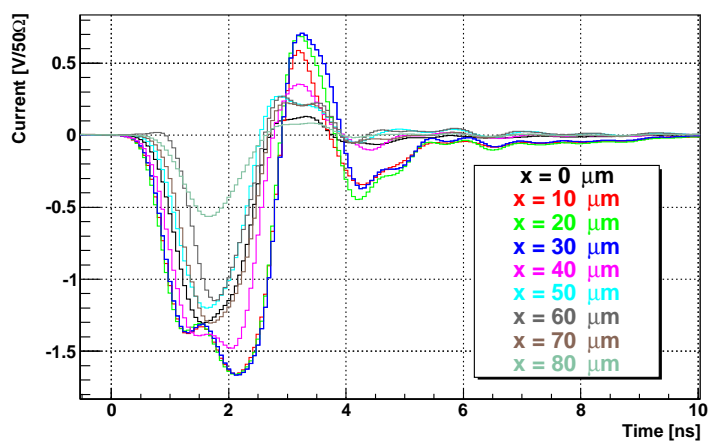

(d)

Figure 19. Waveforms from various positions of illumination over the top surface of an irradiated sensor, after annealing for 20 minutes at $60^{\circ} \mathrm{C}$, moving from the central backside column to an adjacent one either perpendicularly (a) or at $45^{\circ}$ (b). Bias voltages shown were $100 \mathrm{~V}$ (top) or $400 \mathrm{~V}$ (bottom). 


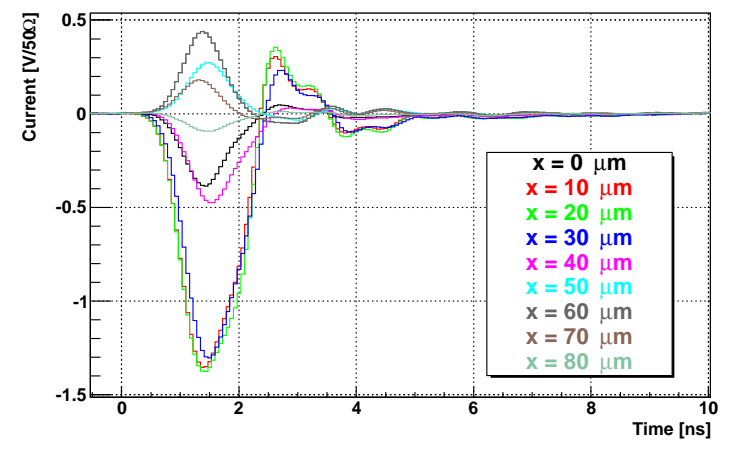

(a)

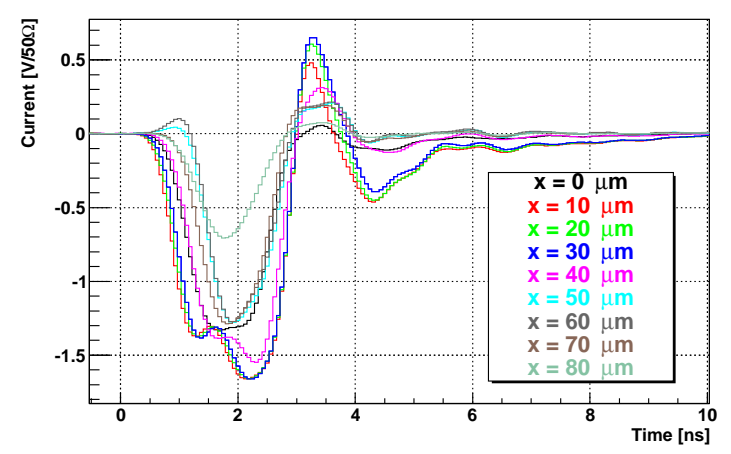

(c)

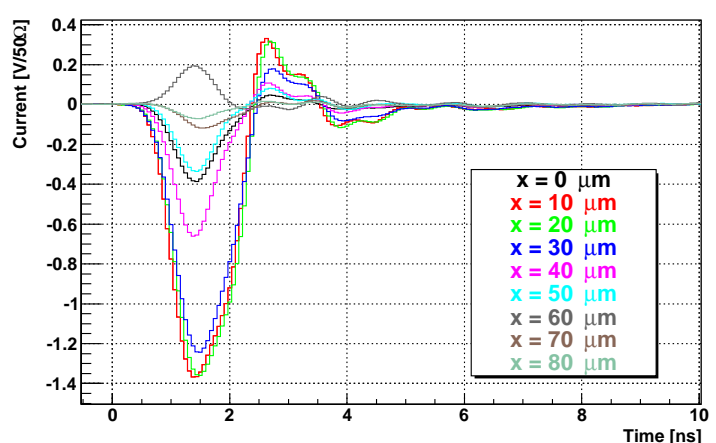

(b)

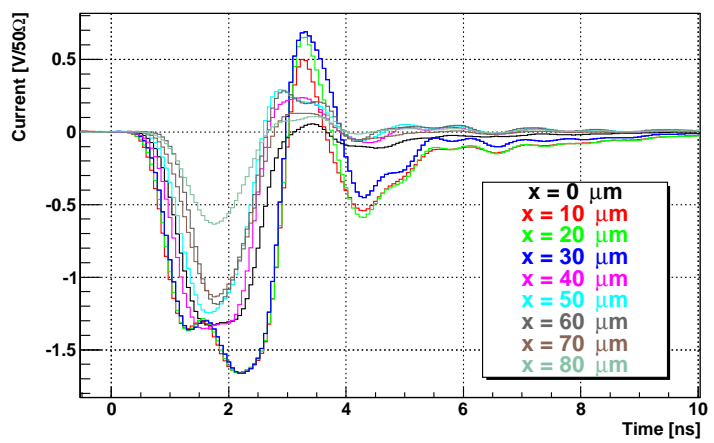

(d)

Figure 20. Waveforms from various positions of illumination over the top surface of an irradiated sensor, after annealing for 300 minutes at $60^{\circ} \mathrm{C}$, moving from the central backside column to an adjacent one either perpendicularly (a) or at $45^{\circ}$ (b). Bias voltages shown were $100 \mathrm{~V}$ (top) or $400 \mathrm{~V}$ (bottom).

$150 \mathrm{~V}$ and $250 \mathrm{~V}$, shorter annealing times lead to higher levels of charge collection. Above $300 \mathrm{~V}$, the charge collected increases due to charge multiplication effects. This charge multiplication effect increases with longer annealing.

The charge collection maps for a unit cell of the device with a bias voltage of $400 \mathrm{~V}$, as shown in figure 22 were examined. After 20 minutes of annealing at $60^{\circ} \mathrm{C}$, the area with the greatest charge collection is the high field regions directly between the n-type and p-type columns. Longer periods of annealing cause the charge collection across the entire active area of the strip to increase proportionally. The waveforms shown previously in figures 19 and 20 show the complete signal is collected well within the $20 \mathrm{~ns}$ time frame required. By comparing the positions of the peaks, it can be seen that there is no change in the time required to collect the charge. Therefore, the increasing collected charge with a longer period of annealing is not due to any change in the charge collection time. Effects of less trapping due to higher carrier velocities and shorter collection times are also not expected to be the cause of the increased charge as the drift velocity has saturated. Therefore the increase in charge collected is attributed to an increase in charge carriers due to a charge multiplication effect.

The electrons in the high electric field directly between $n$ and p-type columns increase their kinetic energy enough to produce further electron-hole pairs. This creates a second, slower current peak. An electric field greater than $10 \mathrm{~V} / \mu \mathrm{m}$ is required for electrons to undergo charge multipli- 


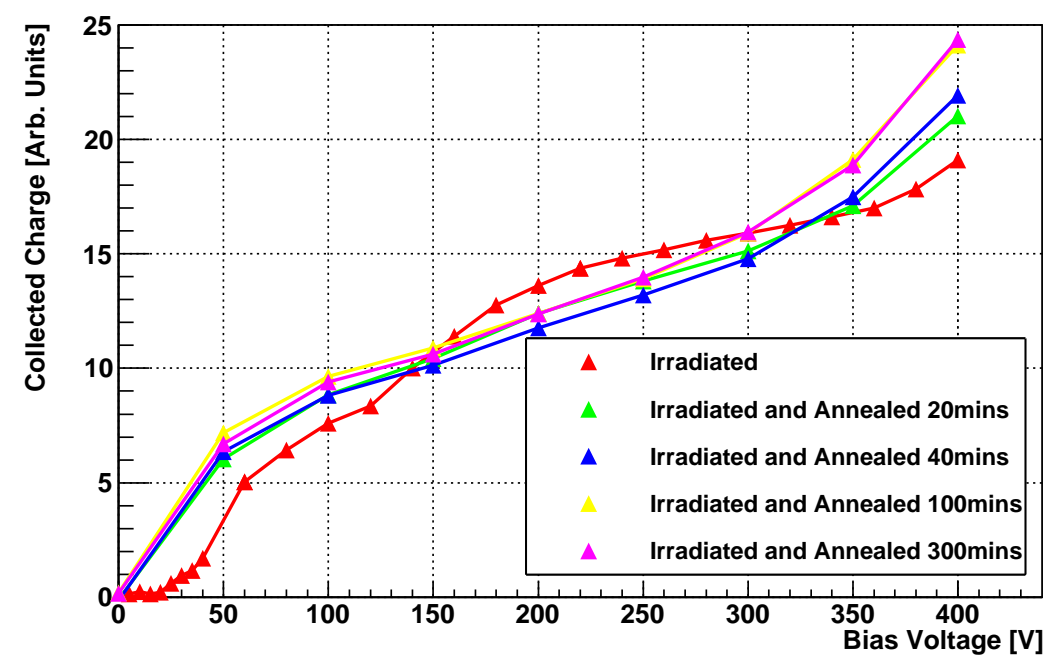

Figure 21. Charge collected versus bias voltage for a MIP-like particle for sensors that were annealed for a range of times.

\section{Charge Collected}

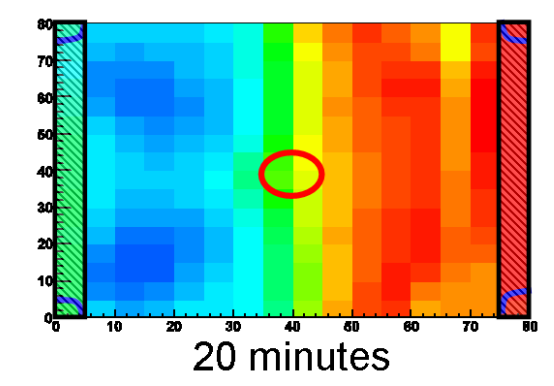

[Arb. Units]
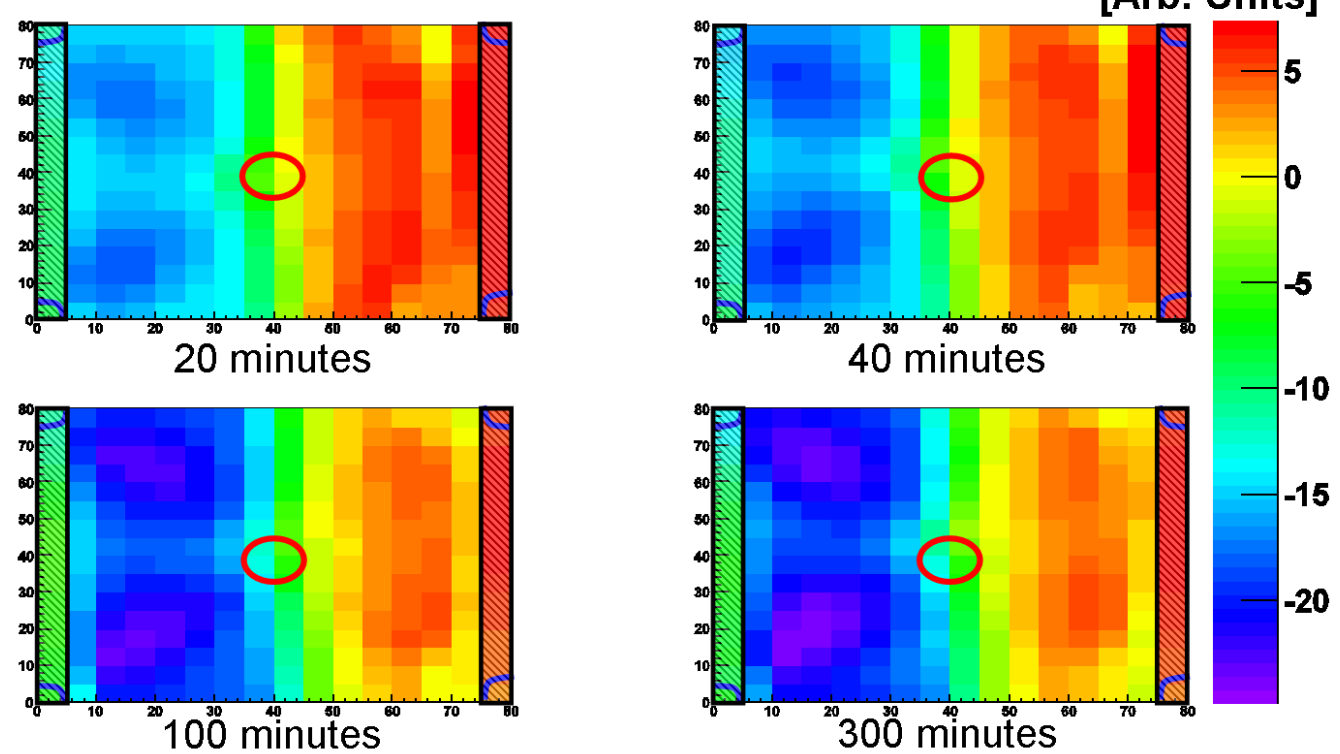

Figure 22. Charge collection maps for a sensor with a bias voltage of $400 \mathrm{~V}$ annealed for 20 minutes, 40 minutes, 100 minutes and 300 minutes. Positions of n-type columns (blue), p-type columns (red) and strips (hatched area) have been indicated.

cation [14]. Due to their electrode structure, 3D devices have larger electric fields than a planar device of equivalent thickness and thus have greater potential for charge multiplication. Holes require a larger electric field to undergo multiplication. Charge multiplication has been observed with conventional charge amplification in irradiated 3D devices [10].

The velocities of charge carriers of the non-irradiated device, the irradiated device and the irradiated device after annealing, shown in figure 23, were compared. For the non-irradiated device, 


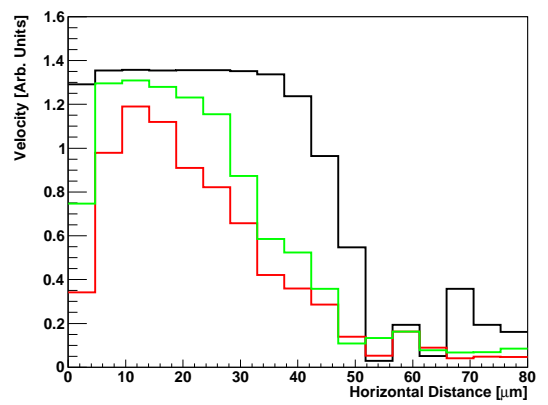

(a) $100 \mathrm{~V}$
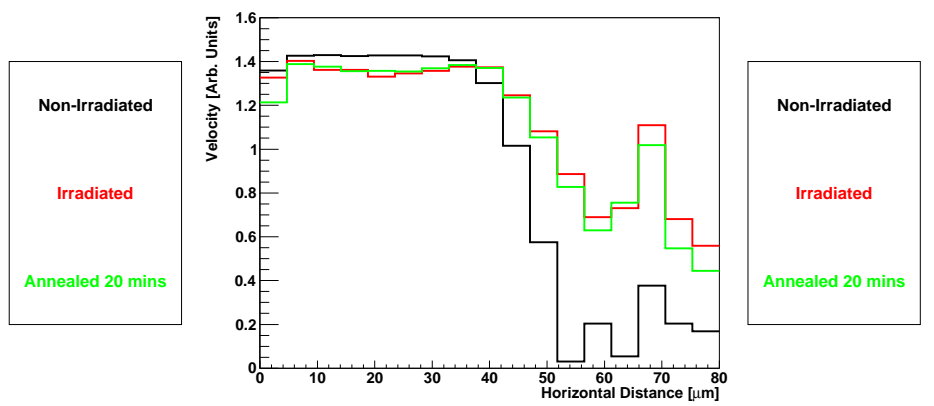

(b) $400 \mathrm{~V}$

Figure 23. Velocity profiles for various illumination positions, moving at $45^{\circ}$ to the strip, for a non-irradiated sensor, an irradiated sensor and an irradiated sensor after annealing for 20 minutes at $60^{\circ} \mathrm{C}$. The nonirradiated sensor was biased to $80 \mathrm{~V}$, and the irradiated and annealed devices were biased to $100 \mathrm{~V}$ in (a) and $400 \mathrm{~V}$ in (b).

$80 \mathrm{~V}$ is a great enough bias to saturate the velocity across the active region. Post-irradiation causes this velocity to not saturate due to distortion of the electric field due to deep defects, even at a slightly greater bias voltage. However, even a short period of annealing provides a beneficial effect and increases the velocity closer to that of the non-irradiated device. With $400 \mathrm{~V}$ bias for the irradiated and annealed devices, it can be seen that the velocity of both the irradiated and annealed detectors approaches that of the non-irradiated detector.

\section{Conclusions}

TCT measurements allow for the velocity of charge carriers within a device to be measured which a standard integrated charge collection technique does not. This enables the magnitude of the electric field to be mapped in a way not possible before. This is because the collected charge saturates at full charge collection whilst the velocity continues to increase with bias voltage.

There is a clear non-uniformity of the sensors prior to irradiation. The areas of greatest carrier velocity and charge collection matches the simulated electric field. While full lateral depletion between the columns occurs at low bias voltages, at approximately $3 \mathrm{~V}$, a uniform carrier velocity between the columns is not achieved until 5 times this value at $20 \mathrm{~V}$. Below $20 \mathrm{~V}$, the greatest velocities of the charge carriers are adjacent to the columns. Both the drift of electrons and holes provide equal contributions to the measured signals. The regions beneath the columns deplete at different rates depending on the type of the column. The region underneath the p-type column depletes with a lower bias voltage than the n-type column.

In addition, charge trapping greatly suppresses the contribution of the holes on the signal produced. This effect is most pronounced around the p-type column. There was no evidence observed of a double-junction around the p-type column. After irradiation there is clear charge multiplication enhancement along the line between columns with a very non-uniform velocity profile in the unit cell of the device. This occurs at bias voltages in excess of the full depletion voltage of the device. The annealing of the detector further enhances these trapping and charge multiplication effects. 


\section{Acknowledgments}

Thanks to the Jozef Stefan Institute in Ljubljana for the polishing of samples, the experimental setup and the irradiation at the reactor. The research leading to these results has received funding from the European Commission under the FP7 Research Infrastructures project AIDA, grant agreement no. 262025. Thanks also to CNM Barcelona for providing the detectors, and the support of the RD50 group.

\section{References}

[1] Z. Li, V. Eremin, N. Strokan and E. Verbitskaya, Investigation of the type inversion phenomena: resistivity and carrier mobility in the space charge region and electrical neutral bulk in neutron irradiated silicon $p^{+}$-n junction detectors, IEEE Trans. Nucl. Sci. 40 (1993) 367.

[2] G. Kramberger et al., Investigation of irradiated silicon detectors by edge-TCT, IEEE Trans. Nucl. Sci. 57 (2010) 2294.

[3] M. Zavrtanik et al., Position sensitive TCT evaluation of irradiated 3D-stc detectors, IEEE Nucl. Sci. Symp. Conf. Rec. 2 (2007) 1318.

[4] G. Pellegrini et al., First double-sided 3-D detectors fabricated at CNM-IMB, Nucl. Instrum. Meth. A 592 (2008) 38.

[5] P. Grenier et al., Test beam results of 3D silicon pixel sensors for the ATLAS upgrade, Nucl. Instrum. Meth. A 638 (2011) 33 [arXiv: 1101.4203].

[6] M. Ravnik and R. Jeraj, Research reactor benchmarks, Nucl. Sci. Eng. 145 (2003) 145.

[7] G. Pellegrini et al., Double sided 3D detector technologies at CNM-IMB, IEEE Nucl. Sci. Symp. Conf. Rec. 2 (2006) 1248.

[8] G. Kramberger, Signal development in irradiated silicon detectors, CERN-THESIS-2001-038, Ph.D. Thesis, Ljubljana University, Ljubljana Slovenia (2001).

[9] G. Lindström et al., Radiation hard silicon detectors - developments by the RD48 (ROSE) collaboration, in 4th Int. Symp. on Development and Application of Semiconductor Tracking Detectors, Hiroshima Japan, 22-25 Mar 2000, Nucl. Instrum. Meth. A 466 (2001) 308.

[10] R.L. Bates et al., Charge collection studies and electrical measurements of heavily irradiated $3 D$ double-sided sensors and comparison to planar strip detectors,

IEEE Trans. Nucl. Sci. 58 (2011) 3370.

[11] D. Pennicard et al., Design, simulation, production and initial characterisation of $3 D$ silicon detectors, Nucl. Instrum. Meth. A 598 (2009) 67.

[12] A. Raighne et al., Precision scans of the Pixel cell response of double sided 3D Pixel detectors to pion and $x$-ray beams, 2011 JINST 6 P05002.

[13] S. Ramo, Currents induced by electron motion, Proc. IRE 27 (1939) 584.

[14] R. Van Overstraeten and H. De Man, Measurement of the ionization rates in diffused silicon pn junctions, Solid-State Electron. 13 (1970) 583. 